\title{
LAS PLANTAS VASCULARES ACUÁTICAS ESTRICTAS Y SU CONSERVACIÓN EN MÉXICO
}

\author{
Arturo Mora-Olivo ${ }^{1,4}$, José Luis Villaseñor ${ }^{2}$ y Mahinda Martínez ${ }^{3}$ \\ ${ }^{1}$ Universidad Autónoma de Tamaulipas, Instituto de Ecología Aplicada, División \\ del Golfo Núm. 356, 87019 Cd. Victoria, Tamaulipas, México. \\ ${ }^{2}$ Universidad Nacional Autónoma de México, Departamento de Botánica, Instituto \\ de Biología; Apdo. postal 70-233, 04510 México, D.F., México. \\ ${ }^{3}$ Universidad Autónoma de Querétaro, Escuela de Biología, Avenida de las \\ Ciencias, Colonia Juriquilla, 76230 Querétaro, Querétaro, México. \\ ${ }^{4}$ Autor para la correspondencia: amorao@uat.edu.mx
}

\section{RESUMEN}

Con base en el análisis de la riqueza y distribución de las hidrófitas vasculares estrictas, se identificaron las entidades federativas más relevantes para la conservación de los humedales de México y su flora asociada. Se registraron 240 especies distribuidas en 106 géneros y 62 familias, incluyendo 227 nativas y 13 introducidas, lo que representa $24 \%$ del total de las plantas acuáticas estimadas para México, incluyendo 8.3\% de endemismo. Los estados que registran la mayor cantidad de especies son Veracruz (145 especies), Jalisco (123), Michoacán (115) y Tamaulipas (113). Un análisis iterativo indica que es necesario involucrar a 13 estados para la conservación de $100 \%$ de la flora acuática nativa.

Palabras clave: conservación, hidrófitas, México, plantas vasculares.

\section{ABSTRACT}

Based on the analysis of richness and distribution of strictly aquatic vascular plants, the states most relevant to wetlands conservation of Mexico and its associated flora were identified. A number of 240 species were identified, distributed in 106 genera and 62 families, including 227 native and 13 introduced. Such richness accounts $24 \%$ of the total estimated of aquatic plants in Mexico and $8.3 \%$ of endemism. The states that have the highest species number are Veracruz (145 species), Jalisco (123), Michoacán 
(115) and Tamaulipas (113). Iterative analysis points out the need to involve 13 states for the conservation of $100 \%$ of the native aquatic flora.

Key words: conservation, hydrophytes, Mexico, vascular plants.

\section{INTRODUCCIÓN}

La conservación de la biodiversidad se ha convertido en una de las prioridades mundiales, tanto para los gobiernos como para los científicos y la sociedad en general (Vane-Wright et al., 1991; Morrone y Crisci, 1992). Se calcula que al menos la mitad de las especies que habitan en el planeta desaparecerán en las próximas décadas (Crisci et al., 2003), especialmente por la destrucción de su hábitat. Particularmente los ambientes acuáticos se cuentan entre los ecosistemas más amenazados (Jain, 1990; Abell, 2001) y en algunas regiones han desparecido hasta en 90\% (Imboden, 1976 citado por Oertli et al., 2002).

Entre las acciones para preservar la biodiversidad, la creación de áreas naturales protegidas ha jugado un papel muy importante (Margules y Pressey, 2000). En la actualidad, la red mundial de estas superficies incluye $11.9 \%$ de toda el área del planeta; sin embargo, la cobertura varía geográficamente y muchas bioregiones y especies no están salvaguardadas o bien representadas (Brooks et al., 2004), sobre todo los humedales y los organismos acuáticos.

En México, como en otras regiones del mundo, los ecosistemas acuáticos han recibido poca atención, no sólo en materia de conservación sino también en cuanto al conocimiento de su biodiversidad y estado de amenaza actual (Olmsted, 1993; Getzner, 2001; Sánchez et al., 2007). En América son pocos los países que se han preocupado por tener un inventario de la flora de sus humedales y conocer su estado de conservación (Lot y Novelo, 1992), tal es el caso de Perú (León y Young, 1996), Costa Rica (Crow, 1993) y Estados Unidos (Reed, 1988). En México, de los $33,185 \mathrm{~km}^{2}$ que cubren los ambientes acuáticos más importantes, distribuidos sobre todo en los ambientes costeros, se calcula que $35 \%$ ha sufrido algún deterioro o ha desaparecido por la deforestación y la expansión de la agricultura, la industria petroquímica y el turismo (Olmsted, 1993; Cervantes, 2007). La Comisión Nacional para el Conocimiento y Uso de la Biodiversidad (CONABIO) seleccionó 110 regiones hidrológicas prioritarias por su alta biodiversidad, uso y/o amenaza; sin embargo $26 \%$ de ellas no cuenta con suficiente información científica al respecto (Arriaga et al., 2000). Por otro lado también existe en el país un Sistema Nacional de Áreas 
Naturales Protegidas (SINAP), que incluye 61 áreas que cubren una superficie de $129,991.01 \mathrm{~km}^{2}$; desafortunadamente sólo unas pocas corresponden o contienen humedales de mayor importancia, como los Pantanos de Centla, Ría Lagartos, Laguna Madre y Cuatrociénegas (Anónimo, 2008; Anónimo, 2012).

La definición de las áreas para su conservación se ha fundamentado en diversos criterios, aunque en la mayoría de las ocasiones se ha usado una estrategia oportunista, que incluye aspectos políticos, sociales, económicos o de disponibilidad (Pressey et al., 1993; Primack et al., 2001). Hoy en día prevalece la idea de usar bases más científicas, como son los parámetros de riqueza, rareza o endemismo de especies; para esto es indispensable contar con un buen conocimiento sobre la taxonomía y distribución geográfica de las especies que se desea proteger (Giangrande, 2003). Se han propuesto diferentes metodologías para la ubicación de sitios prioritarios para la conservación de la biodiversidad y sus ecosistemas (Morrone y Crisci, 1992; Williams et al., 1996; Bonn y Gaston, 2005; Margules y Sarkar, 2007). Entre ellas se pueden citar el método marcador, el análisis de discrepancias, las relaciones filogenéticas, la panbiogeografía, el análisis de parsimonia de endemismos o los métodos iterativos. Varios de ellos usan la distribución de las especies para detectar áreas de mayor diversidad, pues se ha sugerido que la biogeografía juega un papel clave en cuestiones de conservación biológica (Prance, 1994; Morrone y Espinosa, 1998; Whittaker et al., 2005).

Debido a que es mejor el conocimiento de los organismos terrestres que el de los acuáticos, existen pocos antecedentes de estos últimos sobre su uso para proponer sitios de protección de humedales. Entre los pocos estudios destacan el empleo de macroinvertebrados en Inglaterra (Bries, 2004), coleópteros en España (Abellán, 2003), peces costeros en Australia (Fox y Beckley, 2005) y aves acuáticas en Sudáfrica (Turpie, 1995). Los trabajos sobre conservación en los que se han utilizado plantas vasculares acuáticas son aún más escasos; entre ellos se pueden citar los realizados en Australia (Margules et al., 1988; Pressey y Nicholls, 1989) y en Inglaterra (Linton y Goulder, 2000). En México se han hecho propuestas para la conservación de plantas terrestres (Villaseñor et al., 1998, 2003; Lira et al., 2002; Dávila-Aranda et al., 2004), pero no existe algo similar para los vegetales propios de humedales (hidrófitas), quizá debido a que no se cuenta con información suficiente sobre su riqueza y distribución.

El número de especies de plantas con flores acuáticas mexicanas se ha estimado en unas 1,000 (Rzedowski, 1991). Alternativamente, Lot y colaboradores (1993) mencionan la existencia de 747 hidrófitas en México, lo que representa alrededor de $13 \%$ de la flora acuática a nivel mundial; sin embargo, hasta la fecha los 
únicos inventarios conocidos de hidrófitas con carácter nacional (Lot et al., 1986 1999), documentan 118 especies, 45 géneros y 24 familias de angiospermas pertenecientes a familias estrictamente acuáticas. Se han realizado algunos trabajos que describen la diversidad de plantas acuáticas en zonas particulares del país (e.g. Bonilla-Barbosa, 2004; Gutiérrez, 2006; Lot, 2004a, 2004b; Martínez y García, 2001; Novelo, 2006; Ramos y Novelo, 1993). Asimismo, algunas floras regionales (Bajío y regiones adyacentes, Mesoamericana, Valle de México, Nueva Galicia, Valle de Tehuacán-Cuicatlán, Guerrero, etc.) han contribuido a conocer diversos grupos de estos vegetales. Sin embargo, sólo tres de las 32 entidades federativas en que se divide México cuentan con un inventario de ellos publicado de manera exclusiva: Aguascalientes (Siqueiros, 1989), Morelos (Bonilla-Barbosa et al., 2000) y Tamaulipas (Mora-Olivo y Villaseñor, 2007). El escaso conocimiento florístico de los humedales mexicanos es proporcional a los pocos estudios taxonómicos que existen sobre sus plantas; por ejemplo, de 28 familias estrictamente acuáticas presentes en el país, solamente cinco han sido publicadas formalmente, y de 70 géneros con todas sus especies estrictamente acuáticas, únicamente 12 cuentan con tratamiento de su sistemática (Mora-Olivo, datos no publicados).

Es evidente que aún falta mucho por conocer sobre la riqueza y distribución de las plantas acuáticas de México, sobre todo si además de las hidrófitas estrictas se toman en cuenta las especies subacuáticas y tolerantes, que forman un grupo mucho más numeroso. Lamentablemente esta riqueza aún desconocida, se encuentra en grave amenaza por la pérdida de los humedales mexicanos y por la introducción de plantas exóticas que desplazan a muchas especies nativas (Novelo y Martínez, 1989; Mora-Olivo et al., 2008). Es importante entonces comenzar a proponer estrategias para la conservación de las plantas acuáticas de México. Como un paso inicial en este sentido, el presente estudio tiene como objetivo identificar, con base en el análisis de la riqueza y distribución de las especies de hidrófitas vasculares estrictas, las entidades federativas más relevantes para la conservación de los humedales de México y su riqueza florística acompañante.

\section{ÁREA DE ESTUDIO}

Los humedales de México son producto de una conjunción de factores geomorfológicos, hidrológicos y biológicos (Olmsted, 1993). De acuerdo con Lugo y Córdova (2007), el país tiene 22 unidades geomorfológicas continentales, siendo dominantes los relieves montañosos. En consecuencia el sistema hidrológico nacional 
es muy diverso, dividiéndose en 37 regiones y 731 cuencas (Anónimo, 2011), lo que ha favorecido la presencia de distintos humedales que se pueden dividir en marinos, estuarinos, lacustrinos, riverinos y palustrinos (sensu Cowardin et al., 1979).

El área total de los ecosistemas acuáticos mexicanos no se conoce con exactitud. De los ambientes lóticos (con corriente, como ríos y arroyos) sólo se sabe que forman una red hidrológica de $633,000 \mathrm{~km}$ de longitud, pero no existen datos acerca de su superficie (Anónimo, 2011). En el caso de los ambientes acuáticos lénticos (de lento o nulo movimiento, como lagos, lagunas, presas, etc.), se ha estimado que ocupan una extensión de $57,604.2 \mathrm{~km}^{2}$; de ellos, $19,276.5 \mathrm{~km}^{2}$ corresponden a los permanentes y $38,327.7 \mathrm{~km}^{2}$ a los temporales. Por otra parte, $23,877.6 \mathrm{~km}^{2}$ contienen agua salobre o salina y $33,726.6 \mathrm{~km}^{2}$ agua dulce (Anónimo, 1998, Cuadro 1). El Inventario Forestal Nacional (Palacio-Prieto et al., 2000) considera que $31,821 \mathrm{~km}^{2}$ del país constituyen cuerpos de agua y vegetación hidrófila; las entidades federativas con mayor superficie de humedales son Tabasco, Quintana Roo, Campeche, Chiapas, Veracruz, Sinaloa, Tamaulipas y Jalisco (Cuadro 3).

Diferentes comunidades bióticas se han reportado en los humedales mexicanos; entre ellas destacan la vegetación en galería (llamada impropiamente vegetación riparia, bosque ripario y selva alta o mediana riparia), la selva baja inundable, el palmar inundable, la sabana, el manglar, el matorral espinoso inundable, el matorral inerme inundable, el popal, el tular, el carrizal, la vegetación flotante, la vegetación

Cuadro 1. Tipos de humedales en México de acuerdo con su nivel de permanencia (los humedales permanentes poseen agua todo el tiempo, los temporales sólo tienen agua en alguna época del año) y riqueza de especies de hidrófitas estrictas vasculares que contienen. La superficie es la determinada por la Comisión Nacional del Agua (Anónimo, 1998, 2011).

\begin{tabular}{lclr}
\hline Tipo de humedal & Tipo de agua & Superficie $\left(\mathrm{km}^{2}\right)$ & Especies (\%) \\
\hline Humedales permanentes & & & \\
Lagunas y esteros & salobre & $11,386.7$ & $32(13.3)$ \\
Lagos y presas & dulce & $7,889.8$ & $151(62.9)$ \\
Ríos y arroyos & dulce & no disponible & $123(51.2)$ \\
& & & \\
Humedales temporales & & & \\
Lagunas y marismas & salobre & $12,490.9$ & $8(3.3)$ \\
Lagos y presas & dulce & $1,960.1$ & $71(29.6)$ \\
Ríos y arroyos & dulce & no disponible & $32(13.3)$ \\
Pantanos y zonas inundables & dulce & $23,875.7$ & $158(65.8)$ \\
\hline
\end{tabular}


sumergida y la vegetación de pastos marinos o ceibadales (Miranda y Hernández, 1963; Rzedowski, 1978, Sánchez, 1986; Lot y Novelo, 1990; Lot, 2004a). Estas fitocenosis se encuentra distribuidas en el país desde el nivel del mar hasta los 4,000 m de altitud; sin embargo, se concentran principalmente en las zonas donde se combina una alta precipitación y un drenaje deficiente, como la planicie costera del sur de Tamaulipas y de Veracruz, de Tabasco y de Campeche en el Golfo de México o la planicie costera de Nayarit y las zonas lacustres y pantanosas de Michoacán y Jalisco del lado del Océano Pacífico (Rzedowski, 1978).

\section{MÉTODOS}

\section{Conceptos}

En este trabajo se utiliza el concepto de la Convención Ramsar, que define a los humedales como las extensiones de marismas, pantanos y turberas, o superficies cubiertas de agua, sean éstas de régimen natural o artificial, permanentes o temporales, estancadas o corrientes, dulces, salobres o saladas, incluidas las extensiones de agua marina, cuya profundidad en marea baja no exceda de seis metros (Anónimo, 1996). Igualmente, los términos ambiente acuático y ecosistema acuático se emplean como sinónimos de humedal. Por otra parte, una hidrófita o planta acuática es aquella que vive en agua o sobre un substrato que está al menos periódicamente anaerobio debido al exceso de agua (Tiner, 1991) y puede ser estricta, subacuática o tolerante de acuerdo con Lot et al. (1993). Tradicionalmente se ha considerado como hidrófitas estrictas solamente a las plantas de familias donde todos sus integrantes son acuáticos (Lot et al., 1999). Sin embargo, en este caso también se incluyen aquellas especies que a pesar de pertenecer a familias con elementos terrestres, han evolucionado hasta adaptarse morfológica y/o fisiológicamente al medio acuático, teniendo formas de vida emergentes, flotantes o sumergidas. Es importante mencionar que también se consideran algunos elementos leñosos que cumplen con las características de una planta acuática estricta.

Inventario florístico

Con base en la consulta de diversas fuentes de información, como fueron 13,716 ejemplares de herbario (depositados principalmente en ENCB, MEXU, UAMIZ, UAT, TEX y XAL), bases de datos (REMIB, W3TROPICOS, UNIBIO) y literatura especializada (monografías, revisiones taxonómicas y estudios florísticos), se 
obtuvo una lista de las especies de hidrófitas vasculares estrictas en las 32 entidades federativas de México (Fig. 1, Cuadro 3). El catálogo se ordenó por grandes grupos taxonómicos y alfabéticamente por familias, géneros y especies. Se siguió la clasificación de Mickel y Smith (2004) para los helechos y plantas afines, de Brummitt (1992) para las gimnospermas, de Dahlgren et al. (1985) para las monocotiledóneas y de Cronquist (1981) para las dicotiledóneas. Los autores de las especies se abreviaron de acuerdo con Villaseñor et al. (2008).

\section{Distribución}

Para cada especie se registró su presencia a nivel estatal, el tipo de humedal (permanente o temporal, dulceacuícola o salobre, léntico o lótico) y el intervalo altitudinal en que se encuentra. Con base en este último atributo, las mismas se dividieron en dos grupos principales, el primero constituido por las hidrófitas que se distribuyen entre los 0 y los 1000 metros de altitud y el segundo por las que lo hacen por arriba de esta cota. La definición de estos dos grupos sigue algunos criterios utilizados previamente para diferenciar comunidades vegetales tropicales y templadas, tanto acuáticas (Huidobro et al., 2006; Lacoult y Freedman, 2006) como terrestres (Rzedowski, 1978).

Análisis de datos

Para determinar qué estados de México destacan como los más importantes para llevar a cabo estrategias para la conservación de las especies de hidrófitas vasculares estrictas, se utilizó el método iterativo propuesto por Vane-Wright y colaboradores (1991), éste es heurístico y permite de manera fácil determinar sitios prioritarios para la conservación, intentando proteger el mayor número de especies en la menor cantidad de localidades (Pressey y Nicholls, 1989; Margules y Pressey, 2000). Esta metodología consiste simplemente en seleccionar en primer lugar el estado con el mayor número de especies, las cuales se eliminan de la lista original al considerarlas como primera opción. Las no escogidas con esta primera opción constituyen el complemento, el cual es evaluado para elegir nuevamente al estado con el mayor número de especies remanentes, disminuyendo así la lista de plantas que forman el mismo. Cuando dos o más estados tienen el mismo número de especies en el complemento, se elige el que tenga la mayor cantidad total. Si aún continúa el empate entre dos o más entidades federativas, se selecciona el más cercano a alguno previamente escogido. Este proceso se repite continuamente hasta que todas las especies han sido incluidas para alguno de los estados. 
Acta Botanica Mexicana 103: 27-63 (2013)

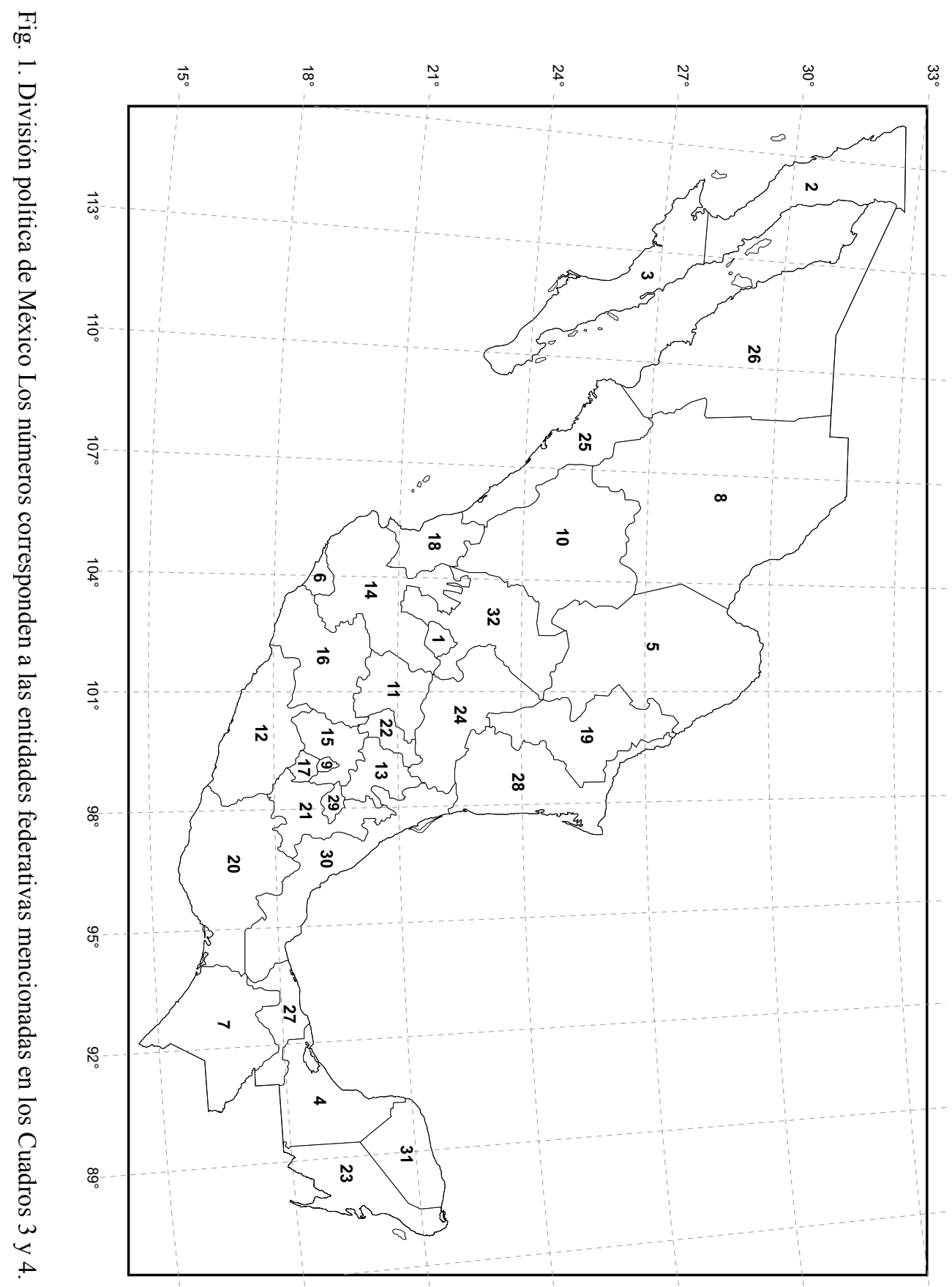




\section{RESULTADOS}

\section{Riqueza florística}

El análisis de la literatura y la consulta del material resguardado en las colecciones consultadas permitieron determinar que los humedales de México contienen 240 especies de hidrófitas vasculares estrictas, distribuidas en 106 géneros y 62 familias; este número incluye 13 especies introducidas (Cuadro 2, Apéndice). El componente principal lo constituyen las angiospermas (221 especies); las monocotiledóneas superan en número a las dicotiledóneas, un patrón no observado entre las plantas terrestres, donde estas últimas son más importantes por su número que las primeras. En la flora de México solamente se registran 18 especies acuáticas estrictas de helechos y plantas afines y una de gimnosperma (Taxodium mucronatum) asociada principalmente a los cuerpos de agua.

Las familias mejor representadas por su riqueza de especies de hidrófitas estrictas son Cyperaceae (25), Alismataceae (18), Lemnaceae (15), Pontederiaceae (15), Nymphaeaceae (13), Potamogetonaceae (11) y Poaceae (10). Los géneros con mayor número de especies son Eleocharis (13), Nymphaea (12), Echinodorus (9), Heteranthera (9), Potamogeton (9), Sagittaria (9) y Ludwigia (8). Por otra parte, los estados que registran la mayor riqueza son Veracruz (145 especies), Jalisco (123), Michoacán (115), Tamaulipas (113), Chiapas (111), Tabasco (111), y Oaxaca (105). La entidad con menor número de hidrófitas estrictas es Tlaxcala (27, Cuadro 3).

Riqueza por tipo de humedal

Existen en México humedales permanentes y temporales (Cuadro 1) y la riqueza encontrada en ambos tipos de cuerpos de agua no es significativamente

Cuadro 2. Riqueza de especies de hidrófitas estrictas vasculares en México y su distribución en los principales grupos taxonómicos.

\begin{tabular}{|c|c|c|c|c|c|c|}
\hline \multicolumn{2}{|l|}{ Grupo } & Familias & Géneros & Especies & $\begin{array}{c}\text { Especies } \\
\text { nativas }\end{array}$ & $\begin{array}{l}\text { Especies } \\
\text { introducidas }\end{array}$ \\
\hline \multicolumn{2}{|c|}{ Helechos y plantas afines } & 6 & 7 & 18 & 17 & 1 \\
\hline \multicolumn{2}{|c|}{ Gimnospermas } & 1 & 1 & 1 & 1 & 0 \\
\hline \multicolumn{2}{|l|}{ Dicotiledóneas } & 30 & 46 & 90 & 82 & 8 \\
\hline \multirow[t]{2}{*}{ Monocotiledóneas } & & 25 & 52 & 131 & 127 & 4 \\
\hline & Total & 62 & 106 & 240 & 227 & 13 \\
\hline
\end{tabular}


Cuadro 3. Estados de México mostrando la superficie de humedales (cuerpos de agua y vegetación hidrófila) reportada en el Inventario Forestal Nacional (Palacio-Prieto et al., 2000), su riqueza total de especies de hidrófitas estrictas vasculares, el número de especies endémicas de México y de especies restringidas (conocidas solamente del estado).

\begin{tabular}{|c|c|c|c|c|c|c|}
\hline Núm. & Estado & $\begin{array}{c}\text { Superficie } \\
\left(\mathrm{km}^{2}\right)\end{array}$ & $\begin{array}{l}\text { Total de } \\
\text { especies }\end{array}$ & $\begin{array}{c}\text { Especies } \\
\text { introducidas }\end{array}$ & $\begin{array}{c}\text { Especies } \\
\text { endémicas }\end{array}$ & $\begin{array}{c}\text { Especies } \\
\text { restringidas }\end{array}$ \\
\hline 1 & Aguascalientes & 15 & 47 & 3 & 3 & 0 \\
\hline 2 & Baja California & 720 & 55 & 4 & 0 & 3 \\
\hline 3 & Baja California Sur & 1,023 & 44 & 2 & 1 & 0 \\
\hline 4 & Campeche & 3,963 & 78 & 2 & 0 & 2 \\
\hline 5 & Coahuila & 466 & 50 & 2 & 0 & 0 \\
\hline 6 & Colima & 155 & 52 & 3 & 2 & 0 \\
\hline 7 & Chiapas & 2,073 & 111 & 4 & 2 & 1 \\
\hline 8 & Chihuahua & 1,066 & 64 & 3 & 6 & 1 \\
\hline 9 & Distrito Federal & 2 & 68 & 5 & 4 & 0 \\
\hline 10 & Durango & 291 & 60 & 2 & 8 & 3 \\
\hline 11 & Guanajuato & 191 & 58 & 3 & 5 & 0 \\
\hline 12 & Guerrero & 597 & 76 & 4 & 4 & 0 \\
\hline 13 & Hidalgo & 114 & 83 & 5 & 4 & 0 \\
\hline 14 & Jalisco & 1,321 & 123 & 5 & 13 & 2 \\
\hline 15 & México & 152 & 92 & 6 & 6 & 2 \\
\hline 16 & Michoacán & 921 & 115 & 5 & 8 & 2 \\
\hline 17 & Morelos & 11 & 80 & 6 & 4 & 0 \\
\hline 18 & Nayarit & 1,068 & 78 & 2 & 5 & 0 \\
\hline 19 & Nuevo León & 133 & 34 & 3 & 0 & 0 \\
\hline 20 & Oaxaca & 956 & 105 & 6 & 4 & 3 \\
\hline 21 & Puebla & 105 & 68 & 4 & 1 & 0 \\
\hline 22 & Querétaro & 23 & 72 & 3 & 6 & 0 \\
\hline 23 & Quintana Roo & 4,397 & 62 & 2 & 0 & 1 \\
\hline 24 & San Luis Potosí & 170 & 53 & 2 & 2 & 0 \\
\hline 25 & Sinaloa & 1,710 & 48 & 2 & 3 & 0 \\
\hline 26 & Sonora & 914 & 67 & 5 & 2 & 0 \\
\hline 27 & Tabasco & 4,531 & 111 & 2 & 0 & 5 \\
\hline 28 & Tamaulipas & 1,613 & 113 & 7 & 2 & 2 \\
\hline 29 & Tlaxcala & 11 & 27 & 1 & 1 & 0 \\
\hline 30 & Veracruz & 1,746 & 145 & 4 & 3 & 2 \\
\hline 31 & Yucatán & 1,296 & 52 & 2 & 0 & 0 \\
\hline \multirow[t]{2}{*}{32} & Zacatecas & 71 & 35 & 3 & 1 & 0 \\
\hline & Total & 31,825 & 240 & 13 & 20 & 29 \\
\hline
\end{tabular}


distinta. Se tienen registradas 197 especies ( $82.1 \%$ del total) en los humedales permanentes y 176 (73.3\%) en los cuerpos de agua temporales. Entre los primeros, los lagos y las presas poseen la mayor riqueza (151 especies), mientras que en los segundos los pantanos y las zonas inundables son los que registran la diversidad más grande (158 especies).

Las plantas de agua salina o salobre están pobremente representadas en la flora estrictamente acuática de México (32 especies en total), destacando principalmente las de agua dulce (224 especies en total). Por otra parte, en los ecosistemas acuáticos lénticos (lagos, lagunas, esteros, pantanos, presas) se registra la mayor riqueza (222 especies); los ambientes lóticos (arroyos, ríos) tienen un menor número de especies (134), de las cuales 18 se localizan estrictamente en cuerpos de agua corriente, como son las nueve de la familia Podostemaceae, además de Alternanthera philoxeroides, Erigeron heteromorphus, Justicia americana, Lobelia purpusii, Ludwigia repens, Myriophyllum sibiricum, Nuphar advena, Sparganium americanum y Taxodium mucronatum.

\section{Distribución altitudinal}

Un número ligeramente mayor de hidrófitas estrictas se conoce de altitudes bajas, es decir, por debajo de los $1000 \mathrm{~m}$. En este intervalo altitudinal se registran 199 especies, mientras que por arriba de esta cota son 147; sin embargo, hay que señalar que 106 se conocen de un intervalo altitudinal amplio, distribuidas por arriba y por debajo de tal cota. Descontando este último número, entonces 93 se conocen solamente de elevaciones inferiores (menos de $1000 \mathrm{~m}$ ) mientras que 41 son características de humedales de montaña (arriba de $1000 \mathrm{~m}$ ).

Entre las hidrófitas conocidas de altitudes bajas, destacan aquellas que viven principalmente en las planicies costeras. Por ejemplo, en total se conocen 48 especies que solamente habitan ambientes costeros, como las características de manglares o los ceibadales (comunidades de pastos marinos) y cuerpos de agua dulce, tal es el caso de Gymnocoronis latifolia y Benjaminia reflexa. Por otra parte, algunas especies que restringen su área a zonas de alta montaña, como Callitriche heterophylla, Isoetes mexicana, Jaegeria glabra, Ranunculus hydrocharoides y $R$. trichopyllus, contienen poblaciones por arriba de los 3,000 m de altitud. Ejemplos de las que registran una distribución altitudinal amplia son Lemna aequinoctialis y Potamogeton pusillus, pues sus poblaciones se conocen desde el nivel del mar hasta más de $3000 \mathrm{~m}$.

Distribución geográfica

Como se indicó previamente, la riqueza de hidrófitas vasculares estrictas está conformada por 227 especies nativas de México y 13 introducidas, pocas de ellas 
mostrando una distribución amplia a nivel nacional. Entre las nativas, solamente Typha domingensis se conoce de los 32 estados en que México está políticamente dividido; por el contrario, 29 especies $(12.1 \%$ del total) restringen su distribución a uno solo, Tabasco (5 especies) y Durango (3) con el mayor número y otras 11 entidades con al menos una de ellas.

Algunas especies tienen registrada hasta la fecha una distribución disyunta dentro del país. Por ejemplo, Echinodorus virgatus solamente se conoce de Nayarit y Veracruz, Lemna trisulca de la Península de Baja California, Distrito Federal, Estado de México e Hidalgo, Myriophyllum pinnatum de Chihuahua y Michoacán, Oserya coulteriana de Sonora a Guerrero y Tamaulipas, Potamogeton natans de Baja California, Distrito Federal y Michoacán, Sagittaria platyphylla de Nuevo León, Tamaulipas y Michoacán y Sparganium eurycarpum de Baja California y el estado de México.

Todos los estados de México registran al menos una de las 13 especies introducidas (Cuadro 3). Entre éstas las más ampliamente distribuidas son Eichhornia crassipes (conocida de 29 entidades) y Nasturtium officinale (de 25); las de distribución más restringida son Alternanthera philoxeroides e Hygrophila polysperma (conocidas solamente de Tamaulipas), Nymphaea capensis (Quintana Roo), Salvinia molesta (Baja California y Sonora) e Hydrilla verticillata (Nuevo León, Sinaloa y Tamaulipas).

Un porcentaje relativamente bajo (8.3\%) de hidrófitas estrictas son endémicas de México (20 especies). Su distribución se ha registrado en 25 estados (Cuadro 3); Jalisco registra el mayor número (13 especies), seguido por Durango y Michoacán (8). De éstas las de distribución geográfica más amplia son Isoetes mexicana y Oserya coulteriana (conocidas de 11 estados); por otra parte, cuatro especies endémicas se conocen como restringidas a uno solo: Eleocharis arsenifera (Chihuahua), Eleocharis reznicekii (Durango), Oserya longifolia (Jalisco) y Tagetes epapposa (Durango).

México es el límite geográfico de 102 hidrófitas estrictas, la mayoría de ellas de afinidad tropical (65 especies, 27.1\%). Entre las especies que registran su distribución más septentrional en nuestro país están Cabomba palaeformis, Crinum erubescens, Echinodorus andrieuxii, Eichhornia heterosperma, Eleocharis mutata, Heteranthera oblongifolia, Hydrocleys parviflora, Nymphaea amazonum y Pontederia rotundifolia. Igualmente, entre las 37 especies de afinidad boreal cuya distribución más meridional está en México se pueden citar algunas que solamente se conocen de estados fronterizos, como Justicia americana (conocida en México solamente de Chihuahua y Coahuila), Lemna turionifera y Pilularia americana (Baja 
California) y otras que se encuentran en zonas más interiores del país, como Elatine brachysperma, Nuphar advena, Potamogeton amplifolius, Sparganium americanum y Wolffiella gladiata. En este grupo de plantas que encuentran en el país sus límites de distribución, destacan especies con distribución disyunta, como Heteranthera oblongifolia, H. seubertiana, Nymphaea novogranatensis, Phyllanthus fluitans y Sagittaria intermedia, que se distribuyen en Sudamérica y además solamente registran una o pocas poblaciones en México.

Estados importantes para la conservación de las hidrófitas vasculares estrictas de México

El análisis iterativo destaca que para lograr con éxito la conservación de las 227 especies de hidrófitas vasculares estrictas nativas de México, será necesario involucrar a 13 estados (Cuadro 4). Con pocas entidades federativas se lograría establecer estrategias para la conservación de un alto porcentaje de ellas; por ejemplo, en cuatro se concentra casi $90 \%$ de toda la flora acuática considerada (Baja California, Michoacán, Tabasco y Veracruz). Sin embargo, todas las entidades reconocidas con el análisis contienen sitios irremplazables, en los que se establecen poblaciones de hidrófitas estrictas restringidas, es decir, conocidas solamente de localidades confinadas a su territorio político (Cuadro 4). De manera particular, por presentar en su área especies endémicas del país México, es principalmente importante la participación de tres estados en las estrategias para su conservación: Chihuahua, Durango y Jalisco.

\section{DISCUSIÓN}

Las 240 especies de hidrófitas vasculares acuáticas estrictas registradas en México representan 32.1\% del total de la flora acuática calculada para México por Lot et al. $(1986,1999)$ y $24 \%$ de la riqueza estimada por Rzedowski (1991). Por lo tanto, es de esperar que en los cuerpos de agua del país donde se registraron estas plantas, se encuentren entre 500 y 700 especies más de plantas subacuáticas y tolerantes de acuerdo con las apreciaciones de estos autores. Sin embargo, estimaciones preliminares revelan que este componente adicional de la flora acuática de México es superior, pues actualmente se conocen cifras por arriba de las 1,000 especies subacuáticas y tolerantes (Mora-Olivo y Villaseñor, datos no publicados).

El número de 240 hidrófitas estrictas en México es relativamente alto cuando se compara con las cifras registradas en otros países. Por ejemplo, para Perú se 
Cuadro 4. Estados considerados prioritarios para la conservación de las especies de hidrófitas estrictas vasculares nativas de México. Las especies restringidas se conocen solamente de un estado.

\begin{tabular}{|c|c|c|c|c|}
\hline Núm. & Estado & $\begin{array}{c}\text { Núm. acumulado } \\
\text { de especies }\end{array}$ & Complemento & $\begin{array}{c}\text { Especies } \\
\text { restringidas }\end{array}$ \\
\hline 1 & Veracruz & 141 & 86 & 2 \\
\hline 2 & Michoacán & 177 & 52 & 2 \\
\hline 3 & Tabasco & 186 & 41 & 5 \\
\hline 4 & Baja California & 197 & 30 & 3 \\
\hline 5 & Jalisco & 206 & 21 & 2 \\
\hline 6 & Oaxaca & 210 & 17 & 2 \\
\hline 7 & Durango & 214 & 13 & 3 \\
\hline 8 & México & 217 & 10 & 2 \\
\hline 9 & Campeche & 220 & 7 & 2 \\
\hline 10 & Coahuila & 223 & 4 & 0 \\
\hline 11 & Chiapas & 225 & 2 & 1 \\
\hline 12 & Querétaro & 226 & 1 & 0 \\
\hline \multirow[t]{2}{*}{13} & Chihuahua & 227 & 0 & 1 \\
\hline & & 227 & 227 & 25 \\
\hline
\end{tabular}

reportan 177 de plantas vasculares asociadas obligadamente a los ambientes acuáticos (León y Young, 1996) y para Chile 54 (Ramírez et al., 1979). Crow (1993) cita además datos para algunos otros países latinoamericanos: Costa Rica (38 especies), Nicaragua (52), Panamá (35) y Ecuador (48). En contraste, México y todos estos países citados son menos diversos que Estados Unidos, ya que Reed (1988) reporta 2,494 especies de plantas catalogadas como obligadas de humedales. Las cifras al parecer apoyan la idea de Crow (1993) de que los ecosistemas acuáticos tropicales son menos diversos que los templados; sin embargo, para considerar válida esta afirmación hay que tomar en cuenta que aún falta mucho por conocer acerca de la biodiversidad de los ambientes acuáticos en países tropicales (Gottgens y Fortney, 2004). Otro aspecto que es necesario subrayar al comparar datos de distintos países, es la diferencia de criterios que existen para definir y clasificar una planta acuática en distintas categorías. Los conceptos de planta acuática estricta (Lot et al., 1999; Mora-Olivo y Villaseñor, 2007) y planta obligada de humedales (Reed, 1988; Tiner, 1991), no necesariamente son equivalentes. Para contrastar adecuadamente la riqueza de la flora acuática de diferentes regiones es recomendable primero uniformizar 
criterios y posteriormente tomar en cuenta otros aspectos, como la superficie que ocupan sus humedales.

La distribución de las especies de hidrófitas estrictas no es uniforme en todo el país. La mayor riqueza se encuentra en los estados que contienen planicie costera, pues allí es donde se localiza la mayor cantidad de humedales (Rzedowski, 1978; Anónimo, 1998; Palacio-Prieto et al., 2000), como por ejemplo el delta de los ríos Pánuco y Tamesí y los Pantanos de Centla. Veracruz es el estado con mayor número de especies; igualmente es el que tiene la mayor extensión de litoral y planicie costera en México (Anónimo, 2007). La escasa representación de hidrófitas estrictas en la flora de algunas entidades del interior del país es reflejo de la menor proporción de humedales en su territorio (Cuadro 2), por ejemplo en Tlaxcala y Zacatecas. Destaca, sin embargo, el Distrito Federal, que a pesar de tener la menor extensión de humedales, posee la densidad de especies por unidad de área más alta, cinco veces mayor que por ejemplo el estado de Veracruz, de acuerdo con los resultados de este estudio. Otros, como Nuevo León o Sinaloa seguramente requieren de mayor exploración en sus humedales, pues su flora acuática dista mucho de ser bien conocida. Es evidente, sin embargo, que la escasa cantidad de humedales en las regiones montañosas del país en comparación con las planicies costeras, ocasiona la baja diversidad en aquellas entidades con territorios de mayor elevación y abrupta orografía; allí es más común encontrar hidrófitas principalmente anuales, registradas especialmente en la temporada de lluvias.

La flora vascular acuática estricta de México está mejor representada en los cuerpos de agua permanentes, como lagos, presas y ríos (82.1\% del total). Sin embargo, los humedales temporales, como charcos y zonas inundables destacan también como zonas de alta riqueza, especialmente a nivel regional, pues en ellos se ha registrado $31.3 \%$ de esta flora. Por ejemplo, en los charcos temporales de Amealco y Huimilpan, en Querétaro, existe una mayor cantidad de especies que en otros cuerpos de agua (Martínez y García, 2001). Igualmente, Williams et al. (2003) al comparar la riqueza de hidrófitas entre diferentes cuerpos de agua en Inglaterra, encontraron que los estanques temporales fueron los más diversos. A pesar de que los humedales temporales pueden permanecer sin agua durante largos períodos de tiempo, las especies adaptadas a vivir en ellos pueden sobrevivir hasta la próxima inundación, debido a la presencia de estructuras reproductoras que soportan la sequía. Ejemplo de este tipo de plantas en México son Callitriche heterophylla, $\mathrm{He}$ teranthera peduncularis, Isoetes mexicana y algunas especies del género Marsilea.

Es notable la preponderancia de plantas viviendo en ambientes con agua dulce $(93.3 \%$ del total). Es bien conocido que las altas concentraciones de sales en el 
agua no permiten la existencia de muchas plantas acuáticas (Sculthorpe, 1985); sin embargo, algunas hidrófitas comunes en aguas dulces llegan a tolerar ciertos niveles bajos de salinidad, como Pachira aquatica o Stuckenia pectinata. Es importante subrayar entonces que la alta riqueza de especies encontrada en los estados con planicies costeras como Jalisco, Tabasco, Tamaulipas y Veracruz, no está definida por plantas de ambientes salobres, sino por especies propias de agua dulce.

La riqueza de especies encontrada en los ambientes lénticos y lóticos es también contrastante. Los primeros registran más de $90 \%$ de la riqueza de especies y los segundos contienen poco más de 50\%. Aunque 115 especies se comparten entre los dos tipos de humedales, un porcentaje muy bajo de ellas (7.5\%) pueden sobrevivir exclusivamente en agua corriente, como las de la familia Podostemaceae, algunas de las cuales tienen una distribución tan restringida que inclusive sólo se conocen de una o dos localidades, tal es el caso de Oserya longifolia (Jalisco) y Vanroyenella plumosa (Jalisco y Oaxaca) (Novelo y Philbrick, 1997).

La mayor riqueza de hidrófitas vasculares estrictas en México se localiza en las zonas de baja altitud, donde hay una mayor profusión y extensión de cuerpos de agua. La escasa cantidad de humedales en regiones montañosas del país ocasiona la baja diversidad de plantas acuáticas, muchas de las cuales son anuales y se localizan solamente en época de lluvias. Por lo tanto, en México la riqueza de hidrófitas es inversamente proporcional a la altitud, un patrón que se ha observado también en otros países, como Brasil (Rolon y Maltchik, 2006), Inglaterra (Jones et al., 2003) o Nepal (Lacoult y Freedman, 2006).

Pocas hidrófitas estrictas muestran una amplia distribución geográfica. La mayoría de ellas (191, 79.6\% del total de especies) se distribuyen en la mitad o menos de los estados en que se divide el país, 29 se conocen en la actualidad solamente de uno. Algunos autores han argumentado que, a nivel global o continental, las plantas acuáticas muestran una vasta repartición espacial (Santamaría, 2002; Les et al., 2003). Sin embargo, a nivel del país tal patrón no es tan evidente, pues pocas hidrófitas estrictas muestran amplia distribución geográfica a lo largo del mismo. En consecuencia, la escala al parecer es importante al momento de explicar las áreas de estas especies, pues muchas de ellas las pueden tener grandes en contexto mundial, aunque a nivel nacional se restringen a pocas localidades. Tal es el caso de Brasenia schreberi, que se registra ampliamente en América, Asia, el occidente de Europa y Australia (Lot et al., 2002), pero en México solamente se le ha encontrado en seis estados (Chiapas, Chihuahua, Jalisco, Michoacán, San Luis Potosí y Tabasco); otros ejemplos son Bolboschoenus robustus y Spartina alterniflora que se conocen de casi todo el Continente Americano, pero en la República Mexicana solamente han 
sido observadas en pocas entidades del Golfo de México (González et al., 2007; Mora-Olivo y Valdés-Reyna, 2011).

El endemismo de hidrófitas acuáticas estrictas en el país es bajo (8.3\%), cifra cercana a lo encontrado previamente por Rzedowski (1991) al discutir toda la flora acuática. Este autor indica que las especies de plantas acuáticas endémicas del país son alrededor de 150; en este trabajo se reportan únicamente 23 de hidrófitas estrictas, por lo que es posible que la mayoría de las consideradas por él como de área restringida sean subacuáticas o tolerantes. En otros países, como Perú y Nueva Zelanda, también se reporta una relativa pobreza de endemismos acuáticos (León y Young, 1996; McGlone et al., 2001).

Quizá el número de especies endémicas no sea sobresaliente, comparado con otros grupos de plantas mexicanas; sin embargo, es importante subrayar que se distribuyen en 24 de los 32 estados del país. La mayoría de estas plantas (16) se encuentran en dos o más entidades, mientras que solamente cuatro de ellas se localizan en uno solo y en dos ocasiones en un solo cuerpo de agua, como sucede con Oserya longifolia que se conoce únicamente del río Purificación en Jalisco (Novelo y Philbrick, 1997) o de Eleocharis arsenifera, que su área se restringe a un humedal de Chihuahua con altas concentraciones de arsénico (González et al., 2005). Es necesario realizar estudios más específicos para entender el por qué de la distribución más amplia de algunos endemismos, o tan restringida de otros.

En México se localiza el límite geográfico de muchas especies acuáticas. De igual manera, en su territorio se registran especies con distribución disyunta, tanto a escala nacional (e.g. Echinodorus virgatus, Potamogeton natans y Oserya coulteriana, ver Apéndice) como continental, por ejemplo Marsilea macropoda (Johnson, 1986) y Phyllanthus fluitans (Lot et al., 1980), ambas conocidas solamente de Sudamérica y México. Su distribución disyunta se explica más que por factores históricos, por la dispersión a larga distancia. Muchas plantas acuáticas mexicanas dependen de aves acuáticas para su diseminación; los registros de varias de estas especies disyuntas a lo largo de las rutas de pájaros migratorios ha sido el principal argumento para apoyar este mecanismo de dispersión como el más importante (Green et al., 2002). Sin embargo, se requiere de trabajo adicional para realmente demostrar la manera como tales plantas han alcanzado la distribución geográfica que muestran en la actualidad. También es posible que varios estados mexicanos y otros países requieran de estudios de campo más intensos, para conocer con mayor precisión la distribución real y actual de las hidrófitas acuáticas estrictas. Algunos registros de especies utilizados en este artículo están basados en ejemplares recolectados desde hace mucho tiempo, por lo que es posi- 
ble que éstas ya se hayan extinguido en su área de distribución original. Tal es el caso de Sparganium eurycarpum que probablemente ya no forme parte de la flora acuática del centro del país como ha sido comentado por otros investigadores (Lot y Zepeda, 2009).

La identificación de 13 estados que ameritan atención especial para llevar a cabo estrategias de conservación de la flora vascular acuática estricta de México es una primera etapa que orientará estudios futuros con mayor detalle. Por ejemplo, es importante contrastar la distribución de las hidrófitas estrictas con las áreas naturales protegidas que existen actualmente en las entidades seleccionadas; seguramente será necesario proponer nuevas reservas en humedales que permitan proteger esta diversidad de plantas acuáticas mexicanas. También es preciso poner especial atención en las especies de área menos amplia, sobre todo las endémicas; muchas de ellas habitan en zonas montañosas, principalmente a lo largo de la Sierra Madre Occidental y en cuerpos de agua temporales, lo que hace su supervivencia muy delicada, por la temporalidad de los ambientes donde viven. Otras especies de distribución restringida se localizan en planicies costeras (a menudo también en charcos temporales), ambientes que son particularmente amenazados sobre todo por las actividades antropogénicas. En consecuencia, se necesita más trabajo para identificar estos charcos temporales, para así evaluar su grado de amenaza y proponer las estrategias de conservación más adecuadas.

La presencia de especies introducidas, muchas de ellas catalogadas como malezas acuáticas, es un fuerte impacto para la flora acuática nativa. Algunas de ellas han llegado recientemente a México a través de los Estados Unidos, como Hydrilla verticillata (Novelo y Martínez, 1989), Hygrophila polysperma (Mora-Olivo et al., 2008), Salvinia molesta (Mora-Olivo y Yatskievych, 2009) y Alternanthera philoxeroides (Mora-Olivo, en prensa). Es imperativo efectuar programas para el control y erradicación de varias de estas plantas; de igual manera, el gobierno mexicano debe de llevar a cabo actividades que prevengan la introducción de nuevas malezas acuáticas, que sin lugar a dudas serán una amenaza para la conservación de la diversidad y endemismo de las hidrófitas mexicanas.

\section{AGRADECIMIENTOS}

Agradecemos al Posgrado en Ciencias Biológicas de la Universidad Nacional Autónoma de México y al Programa de Mejoramiento del Profesorado (PROMEP) de la Universidad Autónoma de Tamaulipas, por el apoyo recibido para realizar los 
estudios de doctorado del primer autor. A diversos especialistas de familias que revisaron la lista florística y/o contribuyeron a la misma: Donald H. Les (varias), George Yatskievych (Salviniaceae), Ishan Al-Shehbaz (Brassicaceae), Jaime Bonilla (Nymphaeaceae), Jerzy Rzedowski (varias), J. Gabriel Sánchez-Ken (Poaceae), Nancy Hensold (Eriocaulaceae), Nelly Diego (Cyperaceae), Patricia Dávila (Poaceae), R. James Hickey (Isoetacee), Robert R. Haynes (varias), Shirley Graham (Lythraceae), Socorro González (Cyperaceae), Thomas F. Daniel (Acanthaceae), Thomas Philbrick (varias). A los revisores anónimos del manuscrito.

\section{LITERATURA CITADA}

Abell, R. 2001. Conservation biology for the biodiversity crisis: a freshwater follow-up. Conserv. Biol. 16: 1435-1437.

Abellán, R. P. 2003. Selección de áreas prioritarias de conservación en la Provincia de Albacete utilizando los coleópteros acuáticos. Tesis de licenciatura. Facultad de Biología, Universidad de Murcia. Murcia, España. Consulta de la versión resumida en internet: http://entomologia.rediris.es/aracnet/e2/11/24/.

Anónimo. 1996. Definición de "humedales" y sistema de clasificación de tipos de humedales. The Ramsar Convention on Wetlands. Dwight Peck, Ramsar. http:// www.ramsar.org/cda/es/ramsar-about-sites-classification-system/main/ramsar/1-36$55 \% 5 \mathrm{E} 21235$ 4000_2

Anónimo. 1998. Inventario de cuerpos de agua y humedales de México, escala 1:250 000. Subgerencia de Saneamiento y Calidad del Agua, Comisión Nacional del Agua. México, D.F., México.

Anónimo. 2007. Agenda estadística de los Estados Unidos Mexicanos. Instituto Nacional de Estadística, Geografía e Informática. Aguascalientes, Aguascalientes. México. $252 \mathrm{pp}$.

Anónimo. 2008. The list of wetlands of international importance. The Secretariat of the Ramsar Convention on Wetlands. Gland, Switzerland. 40 pp.

Anónimo. 2011. Estadísticas del agua en México, edición 2011. Secretaría de Medio Ambiente y Recursos Naturales y Comisión Nacional del Agua. México, D.F., México. 181 pp.

Anónimo. 2012. Áreas naturales protegidas integradas al SINAP. Comisión Nacional de Áreas Naturales Protegidas. México. http://www.conanp.gob.mx/que_hacemos/ sinap.php

Arriaga, L., V. Aguilar y J. Alcocer. 2000. Aguas continentales y diversidad biológica de México. Comisión Nacional para el Conocimiento y Uso de la Biodiversidad. México, D.F., México. 327 pp.

Bonilla-Barbosa, J. 2004. Flora acuática vascular. In: Luna, I., J. J. Morrone y D. Espinosa (eds.). Biodiversidad de la Sierra Madre Oriental. Las Prensas de Ciencias. México, D.F., México. pp. 149-159. 
Bonilla-Barbosa, J., J. A. Viana-Lases y F. Salazar-Villegas. 2000. Listados florísticos de México XX: Flora acuática de Morelos. Instituto de Biología, Universidad Nacional Autónoma de México. México, D.F., México. 29 pp.

Bonn, A. y K. J. Gaston. 2005. Capturing biodiversity: selecting priority areas for conservation using different criteria. Biodiv. Conserv. 14: 1083-1100.

Bries, R. A. 2004. Incorporing connectivity into reserve selection procedures. Biol. Conserv. 103: $77-83$.

Brooks, T. M., M. I. Bakarr, T. Boucher, G. A. B. Da Fonseca, C. Hilton-Taylor, J. M. Hoekstra, T. Moritz, S. Olivieri, J. Parrish, R. L. Pressey, A. S. L. Rodrigues, W. Sechrest, A. Stattersfield, W. Strahm y S. N. Stuart. 2004. Coverage provided by the global protected-area system: is it enough? Bioscience 54: 1081-1091.

Brummitt, R. K. 1992. Vascular plant families and genera. Royal Botanic Gardens. Kew, UK. 804 pp.

Cervantes, M. 2007. Conceptos fundamentales sobre ecosistemas acuáticos y su estado en México. In: Sánchez, O., M. Herzig, E. Peters, R. Márquez y L. Zambrano (eds.). Perspectivas sobre conservación de ecosistemas acuáticos en México. Instituto Nacional de Ecología-Secretaría de Medio Ambiente y Recursos Naturales. México, D.F., México. pp. 37-67.

Cowardin, L. M., V. Carter, F. Focet y E. T. La Roe. 1979. Classification of wetland and deepwater habitats of the United States. Fish and Wildlife Service, U.S. Department of the Interior, Superintendent of Documents, U.S. Government Printing Office. Washington, D.C., USA. 103 pp.

Crisci, J. V., L. Katinas y P. Posadas. 2003. Biodiversity and conservation evaluations. In: Crisci, J. V., L. Katinas y P. Posadas (eds.). Historical biogeography. An introduction. Harvard University Press. Cambridge, USA. pp. 160-173.

Cronquist, A. 1981. An integrated system of classification of flowering plants. Columbia University Press. New York., USA. 1262 pp.

Crow, G. E. 1993. Species diversity in aquatic angiosperms: latitudinal patterns. Aquat. Bot. 44: 229-258.

Dahlgren, R. T. M., H. T. Clifford y P. F. Yeo. 1985. The families of monocotyledons. structure, evolution, and taxonomy. Springer-Verlag. New York., USA. 520 pp.

Dávila-Aranda, P., R. Lira-Saade y J. Valdés-Reyna. 2004. Endemic species of grasses in Mexico: a phytogeographic approach. Biodiv. Conserv. 13: 1101-1121.

Fox, N. J. y L. E. Beckley. 2005. Priority areas for conservation of Western Australian coastal fishes: a comparison of hotspots, biogeographical and complementary approaches. Biol. Conserv. 125: 399-410.

Getzner, M. 2001. Investigating public decisions about protecting wetlands. J. Environ. Manage. 64: 237-246.

Giangrande, A. 2003. Biodiversity, conservation, and the "taxonomic impediment". Aquat. Conserv.: Mar. Freshwat. Ecosyst. 13: 451-459.

González, M. S., J. A. Tena-Flores, M. T. Alarcón-Herrera, E. Flores-Tavizón y N. BarajasAcosta. 2005. An arsenic-tolerant new species of Eleocharis (Cyperaceae) from Chihuahua, Mexico. Brittonia 57: 150-154.

González, M. S., M. González, J. A. Tena, I. L. López, A. A. Reznicek y N. Diego-Pérez. 2007. Sinopsis de Scirpus s.l. (Cyperaceae) para México. Acta Bot. Mex. 82: 15-41. 
Gottgens, J. F. y R. H. Fortney. 2004. Neotropical wetlands: building links among scientists. Wetlands Ecol. Manage. 12: 543-546.

Green, A. J., J. Figuerola y M. I. Sánchez. 2002. Implications of waterbird ecology for the dispersal of aquatic organisms. Acta Oecol. 23:177-189.

Gutiérrez, C. 2006. Lista de especies de plantas acuáticas vasculares de la Península de Yucatán, México. Polibotánica 21: 75-87.

Huidobro, L., J. J. Morrone, J. L. Villalobos y F. Álvarez. 2006. Distributional patterns of freshwater taxa (fishes, crustaceans and plants) from the Mexican transition zone. J. Biogeogr. 33: 731-741.

Jain, S. K. 1990. Conservation of aquatic plants. In: Gopal, B. (ed.). Ecology and management of aquatic vegetation in the Indian subcontinent. Kluwer Academic Publishers. London, UK. pp. 237-241.

Johnson, D. M. 1986. Systematics of the New World species of Marsilea (Marsileaceae). Syst. Bot. Mon. 11: 1-87.

Jones, J. I., W. Li y S. C. Maberly. 2003. Area, altitude and aquatic plant diversity. Ecography 26: 411-420.

Lacoult, P. y B. Freedman. 2006. Relationships between plants and environmental factors along a steep Himalayan altitudinal gradient. Aquat. Bot. 84: 3-16.

León, B. y K. Young. 1996. Aquatic plants of Peru: diversity, distribution and conservation. Biodiv. Conserv. 5: 1169-1190.

Les, D. H., D. J. Crawford, R. T. Kimball, M. L. Moody y E. Landolt. 2003. Biogeography of discontinuously distributed hydrophytes: A molecular appraisal of intercontinental disjunctions. Int. J. Plant Sci. 164: 917-932.

Linton, S. y R. Goulder. 2000. Botanical conservation value related to origin and management of ponds. Aquat. Conserv.: Mar. Freshwat. Ecosyst. 10: 77-91.

Lira, R., J. L. Villaseñor y E. Ortiz. 2002. A proposal for the conservation of the family Cucurbitaceae in Mexico. Biodiv. Conserv. 11: 1699-1720.

Lot, A. 2004a. Flora y vegetación de los humedales de agua dulce en la zona costera del Golfo de México. In: Caso, M., I. Pisanty y E. Ezcurra (comps.). Diagnóstico ambiental del Golfo de México. Instituto Nacional de Ecología-Secretaría de Medio Ambiente y Recursos Naturales. México, D.F., México. pp. 521-553.

Lot, A. 2004b. Fanerógamas acuáticas. In: García-Mendoza, A. J., M. J. Ordóñez y M. Briones-Salas (eds.). Biodiversidad de Oaxaca. Instituto de Biología, Universidad Nacional Autónoma de México-Fondo Oaxaqueño para la Conservación de la Naturaleza-World Wildlife Found. México, D.F., México. pp. 237-248.

Lot, A. y A. Novelo. 1990. Forested wetlands of Mexico. In: Lugo, A. E., M. Brinson y S. Brown (eds.). Ecosystems of the world 15. Forested wetlands. Elsevier. Amsterdam, Netherlands . pp. 287-298.

Lot, A. y A. Novelo. 1992. Afinidades florísticas de las monocotiledóneas acuáticas mesoamericanas. Tulane Studies in Zoology and Botany 1(suppl.): 147-153.

Lot, A., A. Novelo y C. P. Cowan. 1980. Hallazgo en México de una euforbiácea acuática originaria de Sudamérica. Bol Soc. Bot. Méx. 39: 83-89.

Lot, A., A. Novelo y P. Ramírez-García. 1986. Listados florísticos de México V. Angiospermas acuáticas mexicanas 1. Universidad Nacional Autónoma de México. México, D.F., México. 60 pp. 
Lot, A., A. Novelo y P. Ramírez-García. 1993. Diversity of Mexican aquatic vascular plant flora. In: Ramamoorthy, T. P., R. Bye, A. Lot y J. Fa (eds.). Biological diversity of Mexico. Oxford University Press. New York., USA. pp. 577-591.

Lot, A. y C. Zepeda. 2009. Plantas acuáticas. In: Ceballos, G., R. List, G. Garduño, R. López, M. J. Muñozcano, E. Collado y J. Eivin (comps.). La diversidad biológica del Estado de México. Estudio de estado. Gobierno del Estado de México-Secretaría del Medio Ambiente. Toluca de Lerdo, México. pp. 229-241.

Lot, A., A. Novelo, M. Olvera y P. Ramírez-García. 1999. Catálogo de angiospermas acuáticas de México. Hidrófitas estrictas emergentes, sumergidas y flotantes. Cuadernos 33. Instituto de Biología, Universidad Nacional Autónoma de México, México, D.F., México. 161 pp.

Lot, A., F. Ramos y P. Ramírez-García. 2002. Brasenia schreberi J.F. Gmel. (Cabombaceae), un nuevo registro para Chihuahua, México. Bol. Soc. Bot. Méx. 70: 87-88.

Lugo, H. y C. Córdova. 2007. Geomorfología. In: Coll-Hurtado, A. (coord..). Nuevo atlas nacional de México. Instituto de Geografía, Universidad Nacional Autónoma de México. México, D.F., México. NA III 2.

Margules, C. R., A. O. Nicholls y R. L. Pressey. 1988. Selecting networks of reserves to maximize biological diversity. Biol. Conserv. 43: 63-76.

Margules, C. R. y R. L. Pressey. 2000. Systematic conservation planning. Nature 405: 243253.

Margules, C. R. y S. Sarkar. 2007. Systematic conservation. Cambridge University Press. New York, USA. 270 pp.

Martínez, M. y A. García. 2001. Flora y vegetación acuáticas de localidades selectas del estado de Querétaro. Acta Bot. Mex. 54: 1-23.

McGlone, M. S., R. P. Duncan y P. B. Heenan. 2001. Endemism, species selection and the origin and distribution of the vascular plant flora of New Zealand. J. Biogeogr. 28: 199-216.

Mickel, J. T. y A. R. Smith. 2004. The pteridophytes of Mexico. Mem. New York Bot. Gard. 88: 1-1054.

Miranda, F. y E. Hernández-X. 1963. Los tipos de vegetación de México y su clasificación. Bol. Soc. Bot. Méx. 28: 29-179.

Mora-Olivo, A. (en prensa). Primer registro de la maleza acuática Alternanthera philoxeroides (Amaranthaceae) para México. Biotam Nueva Serie.

Mora-Olivo, A. y J. L. Villaseñor. 2007. Diversidad y distribución de la flora vascular acuática de Tamaulipas, México. J. Bot. Res. Inst. Texas 1: 511-527.

Mora-Olivo, A., T. F. Daniel y M. Martínez. 2008. Hygrophila polysperma (Acanthaceae) una maleza acuática registrada por primera vez para la flora mexicana. Rev. Mex. Biodiv. 79: 265-269.

Mora-Olivo, A. y G. Yatskievych. 2009. Salvinia molesta in Mexico. Amer. Fern J. 99(1): 56-58.

Mora-Olivo, A. y J. Valdés-Reyna. 2011. Nota sobre la presencia de Spartina alterniflora Loisel. (Poaceae: Chloridoideae: Cynodonteae) en humedales mexicanos. Acta Bot. Mex. 95:45:50.

Morrone, J. J. y J. V. Crisci. 1992. Aplicación de métodos filogenéticos y panbiogeográficos en la conservación de la biodiversidad biológica. Evol. Biol. 6: 53-66. 
Morrone J. J. y D. Espinosa. 1998. La relevancia de los atlas biogeográficos para la conservación de la biodiversidad mexicana. Ciencia 49: 12-16.

Novelo, A. 2006. Plantas acuáticas de la Reserva de la Biosfera Pantanos de Centla. Espacios Naturales y Desarrollo Sustentable, A.C. Villahermosa, México. 260 pp.

Novelo, A. y M. Martínez. 1989. Hydrilla verticillata (Hydrocharitaceae), problemática maleza acuática de reciente introducción en México. Anales del Inst. Biol. Univ. Nac. Autón. México, Ser. Bot. 58: 97-102.

Novelo, A. y C. T. Philbrick. 1997. Taxonomy of Mexican Podostemaceae. Aquat. Bot. 57: 37-50.

Oertli, B., D. A. Joye, E. Castella, R. Juge, D. Cambin y J.-B. Lachavanne. 2002. Does size matter? The relationship between pond and biodiversity. Biol. Conserv. 104: 59-70.

Olmsted, I. 1993. Wetlands of Mexico. In: Whigham, D., D. Dykyjová y S. Hejný (eds.). Wetlands of the world: inventory, ecology and management. Vol. 1. Kluwer Academic Publishers. London, UK. pp. 637-677.

Palacio-Prieto, J. L., G. Bocco, A. Velásquez, J. F. Mas, F. Takaki-Takaki, L. Luna-González, G. Gómez-Rodríguez, J. López-García, M. Palma M., I. Trejo V., A. Peralta H., J. Prado-Molina, A. Rodríguez-Aguilar, R. Mayorga-Saucedo y F. González M. 2000. La condición actual de los recursos forestales en México: Resultados del Inventario Forestal Nacional 2000. Invest. Geogr., Bol. Inst. Geogr. Univ. Nac. Autón. Méx. 43: 183-203.

Prance, G. T. 1994. The use of phytogeographic data for conservation planning. In: Forey, P. L., C. J. Humphries y R. I. Vane-Wright (eds.). Systematic and conservation evaluation. Systematics Association. Special Volume 50. Clarendron Press. Oxford, UK. pp. 145-163.

Pressey, R. L. y A. O. Nicholls. 1989. Efficiency in conservation evaluation: scoring versus iterative approaches. Biol. Conserv. 50: 199-218.

Pressey, R. L., C. J. Humphries, C. R. Margules, R. I. Vane-Wright y P. H. Williams. 1993. Beyond opportunism: key principles for systematic reserve selection. Trends Ecol. Evol. 8: 124-128.

Primack, R., R. Rozzi y P. Feinsinger. 2001. Establecimiento de áreas protegidas. In: Primack, R., R. Rozzi, P. Feinsinger, R. Dirzo y F. Massardo. Fundamentos de conservación biológica. Perspectivas latinoamericanas. Fondo de Cultura Económica. México, D.F., México. pp. 449-475.

Ramírez, C., M. Romero y M. Riveros. 1979. Habit, habitat, origin and geographical distribution of Chilean vascular hydrophytes. Aquat. Bot. 7: 241-253.

Ramos, L. J. y A. Novelo R. 1993. Vegetación y flora acuáticas de la laguna de Yuriria, Guanajuato, México. Acta Bot. Mex. 25: 61-79.

Reed, P. B. Jr. 1988. National list of plant species that occur in wetlands: 1988 national summary. Biological Report 88. U.S. Fish and Wildlife Service. Washington, D.C., USA. 244 pp.

Rolon, A. S. y L. Maltchik. 2006. Environmental factors as predictors of aquatic macrophyte richness and composition in wetlands of southern Brazil. Hydrobiología 556: 221-231.

Rzedowski, J. 1978. La vegetación de México. Editorial Limusa. México, D.F., México. 432 pp. 
Rzedowski, J. 1991. El endemismo en la flora fanerogámica mexicana: una apreciación analítica preliminar. Acta Bot. Mex. 15: 47-64.

Sánchez S., R. 1986. Vegetación en galería y sus relaciones hidrogeomorfológicas. Ing. Hidr. Méx. 1(3): 70-78.

Sánchez, O., M. Herzig, E. Peters, R. Márquez y L. Zambrano (eds.). 2007. Perspectivas sobre conservación de ecosistemas acuáticos en México. Instituto Nacional de EcologíaSecretaría de Medio Ambiente y Recursos Naturales. México, D.F., México. 294 pp.

Santamaría, L. 2002. Why are most aquatic plants widely distributed? Dispersal, clonal growth and small-scale heterogeneity in a stressful environment. Acta Oecol. 23: 137-154.

Sculthorpe, C. D. 1985. The biology of aquatic vascular plants. Edward Arnold. Ltd. London, UK. 610 pp.

Siqueiros, M. E. 1989. Contribución a la flora acuática y subacuática de Aguascalientes. Universidad Autónoma de Aguascalientes. Aguascalientes, México. 75 pp.

Tiner, R. W. 1991. The concept of a hydrophyte for wetland identification. Bioscience 41: 236-247.

Turpie, J. K. 1995. Priorizing South African estuaries for conservation: a practical example using waterbirds. Biol. Conserv. 74: 175-185.

Vane-Wright, R. I., C. J. Humphries y P. H. Williams. 1991. What to protect? Sytematics and the agony of choice. Biol. Conserv. 55: 235-254.

Villaseñor, J. L., G. Ibarra-Manríquez y D. Ocaña. 1998. Strategies for the conservation of Asteraceae in Mexico. Conserv. Biol. 12: 1066-1075.

Villaseñor, J. L., J. A. Meave, E. Ortiz y G. Ibarra-Manríquez. 2003. Biogeografía y conservación de los bosques tropicales húmedos de México. In: Morrone, J. J. y J. Llorente B. (eds.). Una perspectiva latinoamericana de la biogeografía. Las Prensas de Ciencias, Facultad de Ciencias, Universidad Nacional Autónoma de México. México, D.F., México. pp. 209-216.

Villaseñor, J. L., E. Ortiz y R. Redondo-Martínez. 2008. Catálogo de autores de plantas vasculares de México. $2^{\mathrm{a}}$ ed. Instituto de Biología, Universidad Nacional Autónoma de México y Comisión Nacional para el Conocimiento y Uso de la Biodiversidad. México, D.F., México. 69 pp.

Williams, P., D. Gibson, C. Margules, A. Rebelo, C. Humphries y R. Pressey. 1996. A comparison of richness hotspots, rarity hotspots, and complementarity areas for conserving diversity of British birds. Conserv. Biol. 10: 155-174.

Williams, P., M. Whitfield, J. Biggs, S. Bray, G. Fox, P. Nicolet y D. Sear. 2003. Comparative biodiversity of rivers, streams, ditches and ponds in agricultural landscape in Southern England. Biol. Conserv. 115: 329-341.

Whittaker, R. J., M. B. Araújo, P. J. Jepson, R. J. Ladle, J. E. M. Watson y K. J. Willis. 2005. Conservation biogeography: assessment and prospect. Diversity Distrib. 11: 3-23. 


\section{APÉNDICE}

Lista florística de las hidrófitas estrictas vasculares de México. Con un asterisco $\left(^{*}\right)$ se señalan las especies introducidas. Entre paréntesis se muestra el intervalo altitudinal de cada especie, el número 1 indica el intervalo 0-1000 m s.n.m., el número 2 indica el intervalo 1001-4000 m s.n.m. AGS = Aguascalientes, $\mathrm{BCN}=$ Baja California, BCS = Baja California Sur, CAM = Campeche, CHIS = Chiapas, $\mathrm{CHIH}$ $=$ Chihuahua, $\mathrm{COAH}=$ Coahuila, $\mathrm{COL}=$ Colima, $\mathrm{DF}=$ Distrito Federal, $\mathrm{DGO}=\mathrm{Du}-$ rango, $\mathrm{GTO}=$ Guanajuato, $\mathrm{GRO}=$ Guerrero, $\mathrm{HGO}=$ Hidalgo, $\mathrm{JAL}=$ Jalisco, $\mathrm{MEX}=$ Estado de México, $\mathrm{MICH}=$ Michoacán, $\mathrm{MOR}=$ Morelos, $\mathrm{NAY}=$ Nayarit, $\mathrm{NL}=$ Nuevo León $=, \mathrm{OAX}=$ Oaxaca, $\mathrm{PUE}=$ Puebla, $\mathrm{QRO}=$ Querétaro, $\mathrm{QROO}=$ Quintana Roo, $\mathrm{SLP}=$ San Luis Potosí, SIN $=$ Sinaloa, $\mathrm{SON}=$ Sonora, $\mathrm{TAB}=$ Tabasco, $\mathrm{TAMS}=$ Tamaulipas, TLAX $=$ Tlaxcala, VER $=$ Veracruz, $Y U C=$ Yucatán, $Z A C=$ Zacatecas .

\section{Helechos y plantas afines}

\section{AZOLLACEAE}

Azolla filiculoides Lam. (1) BCN, CHIH, DF, GTO, HGO, JAL, MEX, MICH, MOR, OAX, PUE, QRO, SIN, TAB, VER.

Azolla microphylla Kaulf. $(1,2)$ AGS, BCN, BCS, CHIH, CHIS, DF, GRO, GTO, HGO, JAL, MEX, MICH, MOR, NAY, OAX, PUE, QRO, SIN, SLP, SON, TAB, TAMS, TLAX, VER.

\section{ISOETACEAE}

Isoetes mexicana Underw. (2) CHIH, DF, DGO, GTO, HGO, JAL, MEX, MICH, MOR, NAY, QRO.

\section{MARSILEACEAE}

Marsilea ancylopoda A. Braun $(1,2)$ AGS, CHIH, DGO, GRO, HGO, JAL, MEX, MICH, NAY, OAX, QRO, TAMS.

Marsilea crotophora D.M. Johnson (1) CAM, GRO, TAB, VER.

Marsilea deflexa A. Braun $(1,2)$ JAL, NAY, OAX, PUE, VER.

Marsilea macropoda Engelm ex A. Braun (1) COAH, HGO, NL, TAMS.

Marsilea mollis B.L. Rob. \& Fernald $(1,2)$ AGS, CHIH, DF, CHIS, DGO, GTO, HGO, JAL,

MEX, MICH, MOR, NAY, OAX, PUE, QRO, SLP, SON, TAMS, VER.

Marsilea polycarpa Hook. \& Grev. (1) GRO, JAL, NAY, TAB, VER.

Marsilea vestita Hook. \& Grev. $(1,2)$ AGS, BCN, BCS, CHIH, CHIS, COAH, DF, DGO, GTO, HGO, JAL, MEX, MICH, MOR, NAY, NL, OAX, PUE, QRO, SIN, SLP, SON, TAMS, VER, ZAC.

Pilularia americana A. Braun $(1,2) \mathrm{BCN}$. 
Apéndice. Continuación.

PARKERIACEAE

Ceratopteris pteridoides (Hook.) Hieron. (1) CHIS, GRO, NAY, SIN, TAB, VER.

Ceratopteris thalictroides (L.) Brongn. (1) GRO, NAY, OAX, TAB, TAMS.

\section{PTERIDACEAE}

Acrostichum aureum L. (1) CAM, CHIS, GRO, NAY, OAX, QROO, TAB, TAMS, VER, YUC.

Acrostichum danaeifolium Langsd. \& Fisch. (1) CAM, CHIS, COL, GRO, JAL, MICH, NAY, OAX, QRO, QROO, SLP, TAB, TAMS, VER, YUC.

\section{SALVINIACEAE}

Salvinia auriculata Aubl. (1) CAM, CHIS, GRO, MICH, OAX, QROO, TAB, TAMS, VER, YUC.

Salvinia minima Baker (1) CAM, CHIS, OAX, QROO, TAB, TAMS, VER, YUC.

*Salvinia molesta D.S. Mitch. (1) BCN, SON.

\section{Gimnospermas}

\section{TAXODIACEAE}

Taxodium mucronatum Ten. $(1,2)$ AGS, CHIS, CHIH, COAH, COL, DFE, DGO, GTO, GRO, HGO, JAL, MEX, MICH, MOR, NAY, NLE, OAX, PUE, QRO, SLP, SIN, SON, TAB, TAMS, TLAX, VER, ZAC.

III. Angiospermas

Monocotiledóneas

\section{ALISMATACEAE}

Echinodorus andrieuxii (Hook. \& Arn.) Small $(1,2)$ CAM, CHIS, COL, GRO, JAL, MEX, MICH, MOR, NAY, OAX, QROO, SIN, TAB, VER, YUC.

Echinodorus berteroi (Spreng.) Fassett (1, 2) BCN, BCS, CHIH, COAH, COL, DGO, GRO, JAL, MICH, NAY, NL, OAX, QRO, SLP, SIN, SON, TAMS, VER, YUC.

Echinodorus bolivianus (Rusby) Holm-Niels. (1) CHIS.

Echinodorus cordifolius (L.) Griseb. (1) CAM, TAMS, VER, YUC.

Echinodorus grandiflorus (Cham. \& Schltdl.) Micheli (1) CHIS, DF, TAB, TAMS, VER.

Echinodorus nymphaeifolius (Griseb.) Buchenau (1) CAM, QROO, YUC.

Echinodorus paniculatus Micheli (1) CAM, CHIS, TAB, TAMS, VER.

Echinodorus tenellus (Mart. ex Schult. \& Schult. f.) Buchenau (2) CHIS, VER.

Echinodorus virgatus (Hook. \& Arn.) Micheli (1) NAY, VER. 
Apéndice. Continuación.

Sagittaria demersa J.G. Sm. (2) AGS, CHIH, DGO, HGO, JAL, QRO.

Sagittaria guayanensis Kunth (1) CAM, CHIS, JAL, MEX, NAY, OAX, PUE, TAB, VER.

Sagittaria intermedia Micheli (1) CAM.

Sagittaria lancifolia L. (1, 2) AGS, CAM, CHIS, COL, GRO, JAL, MICH, NAY, OAX, QRO, QROO, TAB, TAMS, VER, YUC.

Sagittaria latifolia Willd. (1, 2) CAM, CHIS, DF, DGO, HGO, JAL, MEX, MICH, MOR, NAY, OAX, PUE, QRO, SIN, TAB, TAMS, TLAX, VER.

Sagittaria longiloba Engelm. ex J.G. Sm. $(1,2)$ AGS, BCN, BCS, DGO, GTO, HGO, JAL, MICH, MOR, NAY, OAX, PUE, QRO, SIN, SON, TAMS.

Sagittaria macrophylla Zucc. (2) DF, HGO, JAL, MEX, MICH, SON, TLAX.

Sagittaria montevidensis Cham. \& Schltdl. $(1,2)$ CHIH, CHIS, COAH, COL, JAL, MICH, NAY, SIN, SON, TAB.

Sagittaria platyphylla (Engelm.) J.G. Sm. $(1,2)$ MICH, NL, TAMS.

AMARYLLIDACEAE

Crinum erubescens Aiton $(1,2)$ BCS, CAM, CHIS, COL, DF, GRO, JAL, MEX, MICH, MOR, NAY, OAX, PUE, QRO, QROO, TAB, TAMS, VER, YUC.

ARACEAE

Pistia stratiotes L. $(1,2)$ BCN, CAM, CHIS, COL, DF, GRO, GTO, HGO, JAL, MEX, MICH, MOR, NAY, OAX, PUE, QROO, SIN, TAB, TAMS, VER, YUC.

ARECACEAE

Acoelorraphe wrightii (Griseb. \& H. Wendl.) H. Wendl. ex Becc. (1) CAM, CHIS, QROO, TAB, TAMS, VER, YUC.

CANNACEAE

Canna glauca L. (1) CAM, CHIS, JAL, MOR, NAY, PUE, QROO, TAB, TAMS, VER, YUC.

CYMODOCEACEAE

Halodule wrightii Asch. (1) BCS, CAM, QROO, SIN, SON, TAB, TAMS, VER, YUC. Syringodium filiforme Kütz. (1) CAM, QROO, TAMS, VER, YUC.

\section{CYPERACEAE}

Bolboschoenus maritimus (L.) Palla $(1,2)$ BCN, BCS, CHIH, COAH, COL, DF, DGO, MEX, MICH, SIN, SON, TAMS.

Bolboschoenus robustus (Pursh) Soják (1) TAB, TAMS, VER.

Cladium jamaicense Crantz $(1,2)$ CAM, CHIH, CHIS, COAH, COL, HGO, NL, OAX, QRO, QROO, SLP, TAB, TAMS, VER, YUC.

Cyperus articulatus L. (1) BCS, CHIS, COL, DF, GRO, GTO, HGO, JAL, MICH, MOR, NAY, NL, OAX, PUE, QROO, SIN, SLP, SON, TAB, TAMS, VER, YUC, ZAC. 
Apéndice. Continuación.

Cyperus gardneri Nees (1) CAM.

Cyperus giganteus Vahl (1) CAM, CHIS, COL, JAL, MICH, OAX, PUE, QROO, TAB, TAMS, VER.

Eleocharis acicularis (L.) Roem. \& Schult. $(1,2)$ AGS, BCN, CAM, CHIH, CHIS, DF, DGO, GRO, GTO, HGO, JAL, MEX, MICH, MOR, OAX, PUE, QRO, QROO, SLP, SON, TAMS, TLAX, VER.

Eleocharis acutangula (Roxb.) Schult. $(1,2)$ CAM, CHIS, DGO, GRO, JAL, NAY, OAX, TAB, TAMS, VER, YUC.

Eleocharis arsenifera S. González, Tena \& T. Alarcón (2) CHIH.

Eleocharis cellulosa Torr. (1) CAM, CHIS, COAH, GRO, JAL, MEX, NL, QROO, TAB, TAMS, VER, YUC.

Eleocharis densa Benth. $(1,2)$ AGS, DGO, GTO, HGO, JAL, MEX, MICH, MOR, PUE, QRO, VER.

Eleocharis elegans (Kunth) Roem. \& Schult. $(1,2)$ CAM, CHIS, COL, GRO, GTO, HGO, JAL, MEX, MICH, MOR, NAY, OAX, PUE, QRO, QROO, SIN, SLP, SON, TAB, TAMS, VER.

Eleocharis interstincta (Vahl) Roem. \& Schult. (1) CAM, CHIH, CHIS, COAH, COL, HGO, MOR, OAX, QRO, QROO, TAB, TAMS, VER, YUC.

Eleocharis mutata (L.) Roem. \& Schult. $(1,2)$ CAM, CHIS, COL, DGO, GRO, JAL, MEX, NAY, OAX, QROO, TAB, TAMS, VER, YUC.

Eleocharis obtusetrigona (Lindl. \& Nees) Steud. (1, 2) TAMS, VER.

Eleocharis plicarhachis Griseb. \& Svenson (1) CHIS, TAB, VER, YUC.

Eleocharis quadrangulata (Michx.) Roem. \& Schult. (1) DGO, JAL, MICH, NAY, TAMS.

Eleocharis reznicekii S. Elizondo, D.J. Rosen, R.Carter \& P.Peterson (2) DGO.

Eleocharis yecorensis Roalson $(1,2)$ DGO, JAL, SON.

Oxycaryum cubense (Poepp. \& Kunth) Palla $(1,2)$ CAM, CHIS, GRO, GTO, JAL, MICH, NAY, QROO, TAB, TAMS, VER, YUC.

Schoenoplectus acutus (Muhl. ex Bigelow) Á. Löve \& D. Löve $(1,2)$ AGS, BCN, CHIH, COAH, MICH, NL, SON, ZAC.

Schoenoplectus americanus (Pers.) Volkart ex Schinz \& R. Keller $(1,2)$ BCN, CHIH, CHIS, COAH, COL, DF, GTO, HGO, JAL, MEX, MICH, MOR, NL, OAX, PUE, SIN, SON, TAMS, TLAX, VER.

Schoenoplectus californicus (C.A. Mey.) Soják $(1,2)$ BCN, BCS, CHIH, CHIS, COAH, COL, DF, DGO, GTO, HGO, JAL, MEX, MICH, MOR, NL, OAX, PUE, QRO, SLP, SON, TAMS, TLAX, VER.

Schoenoplectus pungens (Vahl) Palla $(1,2)$ BCN, BCS, COAH, GTO, JAL, MICH, SON, TAMS, VER.

Schoenoplectus tabernaemontani (C.C. Gmel.) Palla $(1,2)$ CAM, CHIH, CHIS, COAH, COL, DF, DGO, JAL, MEX, MICH, MOR, NAY, NL, OAX, PUE, QRO, QROO, SON, TAB, TAMS, TLAX, VER, YUC. 
Apéndice. Continuación.

ERIOCAULACEAE

Eriocaulon melanocephalum Kunth (1) TAB.

HYDROCHARITACEAE

*Egeria densa Planch. $(1,2)$ AGS, DF, HGO, JAL, MEX, MOR, NAY, OAX, QRO, VER, YUC.

Halophila decipiens Ostenf. (1) VER.

Halophila engelmannii Asch. (1) TAM, VER.

*Hydrilla verticillata (L.f.) Royle (1) NL, SIN, TAM.

Limnobium laevigatum (Humb. \& Bonpl. ex Willd.) Heine $(1,2)$ DF, HGO, MEX, MICH, SLP, TAB, VER.

Thalassia testudinum Banks \& Sol. ex K.D. Koenig (1) CAM, QROO, TAMS, VER, YUC. Vallisneria americana Michx. (1, 2) CAM, MEX, MOR, QROO, TAB, TAMS, VER.

JUNCAGINACEAE

Triglochin maritima L. (1, 2) BCN, BCS, CHIH, DGO.

Triglochin mexicana Kunth (2) BCS, DF, GTO, MEX, MICH, PUE, TLAX.

\section{LEMNACEAE}

Lemna aequinoctialis Welw. $(1,2)$ AGS, BCN, BCS, CAM, CHIS, COAH, COL, GRO, GTO, HGO, JAL, MEX, MICH, MOR, NAY, OAX, QRO, QROO, SLP, SIN, SON, TAB, TAMS, VER, YUC, ZAC.

Lemna gibba L. (1, 2) AGS, BCN, BCS, CHIH, COAH, DF, DGO, GTO, HGO, JAL, MEX, MICH, MOR, OAX, PUE, QRO, SLP, SON, TAMS, TLAX, VER, ZAC.

Lemna minuta Kunth $(1,2)$ AGS, BCN, BCS, CAM, CHIH, CHIS, COAH, DF, GRO, GTO, HGO, JAL, MEX, MICH, MOR, NL, OAX, PUE, QRO, SLP, SON.

Lemna obscura (Austin) Daubs $(1,2)$ AGS, CHIS, DF, HGO, JAL, MEX, MICH, MOR, PUE, TAB.

Lemna trisulca L. (2) BCN, BCS, DF, HGO, MEX.

Lemna turionifera Landolt (2) BCN.

Lemna valdiviana Phil. $(1,2) \mathrm{BCN}, \mathrm{BCS}, \mathrm{CHIH}, \mathrm{COAH}, \mathrm{COL}$, DF, DGO, GRO, HGO, JAL, MEX, MICH, MOR, OAX, QROO, TAB.

Spirodela intermedia W. Koch (1) CHIS, TAB.

Spirodela polyrhiza (L.) Schleid. $(1,2)$ CHIH, CHIS, COL, DF, GRO, HGO, JAL, MEX, MICH, NAY, OAX, QRO, SIN, SON, TAB, TAMS, VER, YUC.

Wolffia brasiliensis Wedd. (1, 2) AGS, CAM, CHIS, DF, HGO, JAL, MEX, MICH, MOR, OAX, PUE, SLP, TAB, TAMS, YUC, ZAC.

Wolffia columbiana H. Karst. (1, 2) AGS, DF, HGO, JAL, MEX, MICH, MOR, OAX, PUE, QRO, SIN, TAB, TAMS, TLAX, YUC.

Wolfiella gladiata (Hegelm.) Hegelm. (2) DF, HGO, MEX.

Wolffiella lingulata (Hegelm.) Hegelm. (1, 2) CHIS, DF, GTO, HGO, JAL, MEX, MICH, TAB, TAMS, VER. 
Apéndice. Continuación.

Wolffiella oblonga (Phil.) Hegelm. $(1,2)$ DF, HGO, MEX, TAB, VER.

Wolffiella welwitschii (Hegelm.) Monod (1) CHIS, MOR, TAB.

\section{LILAEACEAE}

Lilaea scilloides (Poir.) Hauman $(1,2)$ AGS, BCN, CHIH, DGO, GRO, GTO, HGO, JAL, MEX, MICH, MOR, PUE, QRO, TLAX, ZAC.

\section{LIMNOCHARITACEAE}

Hydrocleys nymphoides (Willd.) Buchenau (1) VER.

Hydrocleys parviflora Seub. (1) CHIS, VER.

Limnocharis flava (L.) Buchenau (1) CAM, CHIS, GRO, NAY, OAX, SIN, SON, TAB, VER.

Limnocharis laforestii Griseb. (1) CHIS, NAY, OAX, VER.

MARANTACEAE

Thalia geniculata L. (1, 2) CAM, CHIS, COL, GRO, JAL, MICH, MOR, NAY, OAX, PUE, QROO, SIN, SLP, TAB, TAMS, VER, YUC.

\section{MAYACACEAE}

Mayaca fluviatilis Aubl. (1, 2) CHIS, JAL, MEX, MICH, NAY, TAB, VER.

\section{NAJADACEAE}

Najas guadalupensis (Spreng.) Magnus (1, 2) AGS, BCN, BCS, CAM, CHIH, CHIS, COAH, COL, DF, DGO, GRO, GTO, HGO, JAL, MEX, MICH, MOR, NAY, NL, OAX, PUE, QRO, SIN, SLP, SON, TAB, TAMS, VER, YUC.

Najas marina L. $(1,2)$ AGS, BCN, BCS, CAM, COAH, DGO, GRO, MICH, MOR, NAY, NL, QROO, SIN, SON, TAMS, VER.

Najas wrightiana A. Braun (1) CAM, CHIS, QROO, TAB, TAMS, VER, YUC.

\section{ORCHIDACEAE}

Habenaria repens Nutt. $(1,2)$ CAM, CHIS, GRO, HGO, JAL, MICH, QRO, TAB, TAMS, VER.

\section{POACEAE}

Hymenachne amplexicaulis (Rudge) Nees $(1,2)$ CAM, CHIS, COL, GRO, JAL, MICH, NAY, NL, OAX, QROO, SIN, TAB, TAMS, VER.

Leersia hexandra Sw. (1, 2) CAM, CHIS, COAH, DF, DGO, GTO, HGO, JAL, MEX, MICH, MOR, NAY, OAX, PUE, TAB, TAMS, VER, ZAC.

Leptochloa aquatica Scribn. \& Merr. (1) COL, GRO, JAL, MICH, MOR, NAY, SIN, ZAC. Luziola fluitans (Michx.) Terrell \& H. Rob. $(1,2)$ AGS, CHIH, GTO, HGO, JAL, MEX, MICH, NAY, QRO, ZAC. 
Apéndice. Continuación.

Panicum elephantipes Nees ex Trin. $(1,2)$ COL, DF, GTO, HGO, JAL, MEX, MICH, QROO, TAB, ZAC.

Paspalidium geminatum (Forssk.) Stapf $(1,2)$ AGS, BCN, BCS, CAM, CHIS, COAH, COL, DGO, GRO, GTO, HGO, JAL, MEX, MICH, MOR, NAY, NL, OAX, PUE, QRO, QROO, SIN, SLP, SON, TAB, TAMS, TLAX, VER, YUC, ZAC.

Paspalum acuminatum Raddi $(1,2)$ MICH, MOR, PUE, TAB, TAMS, VER.

Paspalum longicuspe Nash $(1,2)$ CHIS, GRO, JAL, MICH, MOR, NAY, OAX, SIN, VER.

Paspalum repens P.J. Bergius (1) CAM, CHIS, GRO, TAB, VER.

Spartina alterniflora Loisel. (1) TAMS, VER.

PONTEDERIACEAE

Eichhornia azurea (Sw.) Kunth (1) COL, GRO, JAL, MICH, OAX, SON, TAB, VER.

*Eichhornia crassipes (Mart.) Solms $(1,2)$ AGS, BCN, CAM, CHIS, COAH, COL, DF, DGO, GRO, GTO, HGO, JAL, MEX, MICH, MOR, NAY, NL, OAX, PUE, QRO, QROO, SIN, SLP, SON, TAB, TAMS, VER, YUC, ZAC.

Eichhornia heterosperma Alexander (1) CHIS, TAB.

Eichhornia paniculata (Spreng.) Solms (1) OAX, VER.

Heteranthera dubia (Jacq.) MacMill. $(1,2)$ CAM, CHIH, COAH, DGO, HGO, JAL, NAY, OAX, QROO, SLP, SON, TAB, TAMS, VER.

Heteranthera limosa (Sw.) Willd. (1, 2) AGS, CHIH, CHIS, COAH, COL, DF, DGO, GRO, GTO, HGO, JAL, MEX, MICH, MOR, NAY, OAX, PUE, QRO, QROO, SIN, SLP, SON, TAMS, VER, YUC, ZAC.

Heteranthera mexicana S. Watson (1) COAH, QRO, QROO, TAMS, YUC.

Heteranthera oblongifolia C. Mart. ex Roem. \& Schult. (1) OAX.

Heteranthera peduncularis Benth. $(1,2)$ AGS, CHIH, DF, DGO, GTO, HGO, JAL, MEX, MICH, MOR, NAY, OAX, PUE, QRO, SLP, VER, ZAC.

Heteranthera reniformis Ruiz \& Pav. $(1,2)$ CHIS, COAH, COL, GTO, HGO, JAL, MEX, MICH, MOR, NAY, OAX, PUE, QRO, SLP, TAB, TAMS, VER.

Heteranthera rotundifolia (Kunth) Griseb. $(1,2)$ AGS, CHIH, CHIS, COAH, DGO, GRO, GTO, HGO, JAL, MEX, MICH, MOR, NAY, OAX, PUE, QRO, SIN, SLP, SON, TAMS, VER, YUC, ZAC.

Heteranthera seubertiana Solms (1) CHIS, QROO, YUC.

Heteranthera spicata C. Presl (1) JAL.

Pontederia rotundifolia L.f. (1) GRO, MOR, OAX, TAB, VER.

Pontederia sagittata C. Presl $(1,2)$ CAM, CHIS, GRO, MICH, OAX, PUE, QROO, TAB, VER.

POTAMOGETONACEAE

Potamogeton amplifolius Tuck. (2) MICH.

* Potamogeton crispus L. (1, 2) CHIH, GRO, JAL, MEX, MICH, MOR, PUE, SLP.

Potamogeton diversifolius Raf. (2) AGS, CHIH, COAH, GTO, JAL, MICH, NAY, QRO, SLP, SON. 
Apéndice. Continuación.

Potamogeton foliosus Raf. $(1,2)$ AGS, BCN, BCS, CHIH, COL, DF, DGO, GRO, GTO, HGO, JAL, MEX, MICH, MOR, OAX, PUE, QRO, SLP, SON, TAB, TAMS, VER, ZAC.

Potamogeton illinoensis Morong $(1,2) \mathrm{BCN}, \mathrm{BCS}, \mathrm{CAM}, \mathrm{CHIH}, \mathrm{CHIS}, \mathrm{COAH}, \mathrm{DF}, \mathrm{DGO}$, HGO, JAL, MEX, MICH, MOR, NAY, NL, QRO, QROO, SLP, TAB, TAMS, VER.

Potamogeton natans L. $(1,2) \mathrm{BCN}, \mathrm{DF}, \mathrm{MICH}$.

Potamogeton nodosus Poir. $(1,2)$ AGS, BCN, CAM, CHIH, CHIS, COAH, COL, DF, DGO, GTO, HGO, JAL, MEX, MICH, NAY, NL, OAX, PUE, QRO, SLP, SON, TAB, TAMS, TLAX, VER, ZAC.

Potamogeton praelongus Wulfen (2) MEX.

Potamogeton pusillus L. (1, 2) AGS, BCN, CHIH, CHIS, COL, DF, DGO, GTO, HGO, JAL, MEX, MICH, MOR, PUE, QRO, SLP, SON, TAMS, VER, ZAC.

Stuckenia pectinata (L.) Börner $(1,2)$ BCN, BCS, CAM, COAH, COL, DF, GRO, GTO, HGO, JAL, MEX, MICH, MOR, NAY, NL, OAX, PUE, QRO, SLP, SIN, SON, TAMS, TLAX, VER.

Stuckenia striata (Ruiz \& Pav.) Holub (1, 2) COAH, GRO, GTO, JAL, MICH, NAY, QRO, SON, TAMS, VER.

\section{RUPPIACEAE}

Ruppia didyma Sw. ex Wikstr. (1) COL, JAL, NAY, OAX, SIN, YUC.

Ruppia maritima L. $(1,2)$ BCN, BCS, CAM, CHIS, COAH, COL, DF, GRO, JAL, MEX, MOR, NAY, OAX, PUE, QROO, SIN, SLP, SON, TAB, TAMS, VER, YUC.

\section{SPARGANIACEAE}

Sparganium americanum Nutt. (2) DGO.

Sparganium eurycarpum Engelm. (2) BCN, MEX.

\section{TYPHACEAE}

Typha domingensis Pers. $(1,2)$ AGS, BCN, BCS, CAM, CHIH, CHIS, COAH, COL, DF, DGO, GRO, GTO, HGO, JAL, MEX, MICH, MOR, NAY, NL, OAX, PUE, QRO, QROO, SLP, SIN, SON, TAB, TAMS, TLAX, VER, YUC, ZAC.

Typha latifolia L. (2) AGS, BCN, BCS, CAM, CHIH, CHIS, COAH, COL, DF, DGO, HGO, JAL, MEX, MICH, MOR, NAY, NL, OAX, PUE, QRO, SIN, SON, TAB, TAMS, TLAX, VER.

\section{ZANNICHELLIACEAE}

Zannichellia palustris L. $(1,2)$ AGS, BCN, BCS, CHIH, COAH, DF, GTO, HGO, JAL, MEX, MICH, MOR, NL, OAX, PUE, QRO, SIN, SLP, SON, TAMS, VER, ZAC.

\section{ZOSTERACEAE}

Phyllospadix scouleri Hook. (1) BCN, BCS. 
Apéndice. Continuación.

Phyllospadix torreyi S. Watson (1) BCN, BCS.

Zostera marina L. (1) BCN, BCS, SIN, SON.

Dicotiledóneas

ACANTHACEAE

*Hygrophila polysperma (Roxb.) T. Anderson (1) TAMS.

Justicia americana Vahl (1) CHIH, COAH.

AMARANTHACEAE

*Alternanthera philoxeroides (Mart.) Griseb. (1) TAMS.

ANNONACEAE

Annona glabra L. (1) CAM, COL, GRO, JAL, NAY, OAX, QROO, TAB, TAMS, VER, YUC.

APIACEAE

Berula erecta (Huds.) Coville $(1,2)$ AGS, BCN, CHIH, CHIS, COAH, DF, DGO, GTO, HGO, JAL, MEX, MICH, MOR, NL, OAX, PUE, QRO, SON, TLAX, VER, ZAC.

Hydrocotyle ranunculoides L. f. (2) AGS, BCN, BCS, CHIH, COAH, DF, DGO, GTO, HGO, JAL, MEX, MICH, MOR, OAX, PUE, QRO, SLP, SON, TLAX, VER, ZAC.

Lilaeopsis schaffneriana (Schltdl.) J. M. Coult. \& Rose $(1,2)$ AGS, CHIH, COAH, DF, DGO, HGO, JAL, MEX, MICH, MOR, NL, PUE, QRO, SLP, SON, TAMS, TLAX, VER, ZAC.

ASTERACEAE

Erigeron heteromorphus B.L. Rob. (1) QRO, SLP.

Gymnocoronis latifolia Hook \& Arn. (1) CAM, CHIS, NAY, TAB, TAMS, VER.

Hydropectis aquatica (S. Watson) Rydb. (2) CHIH, DGO.

Jaegeria glabra (S. Watson) B.L. Rob. (2) AGS, CHIH, DF, DGO, GTO, HGO, JAL, MEX, $\mathrm{MICH}, \mathrm{QRO}$.

Jaegeria purpurascens B.L. Rob. (2) DGO, JAL, QRO.

Olivaea tricuspis Sch. Bip. (2) AGS, GTO, JAL, MEX, MICH.

Tagetes epapposa B. L. Turner (2) DGO.

BOMBACACEAE

Pachira aquatica Aubl. $(1,2)$ CAM, CHIS, GRO, MICH, NAY, OAX, PUE, QROO, SIN, TAB, VER, YUC.

BRASSICACEAE

*Nasturtium officinale R. Br. $(1,2)$ AGS, BCN, BCS, CHIH, CHIS, COAH, COL, DF, DGO, 
Apéndice. Continuación.

GRO, GTO, HGO, JAL, MEX, MICH, MOR, NL, OAX, PUE, QRO, SON, TAMS, TLAX, VER, ZAC.

\section{CABOMBACEAE}

Brasenia schreberi J. F. Gmel. (2) CHIH, CHIS, JAL, MICH, SLP, TAB.

Cabomba palaeformis Fassett $(1,2)$ CAM, CHIS, MEX, MOR, OAX, QROO, SLP, TAB, TAMS, VER.

\section{CALLITRICHACEAE}

Callitriche heterophylla Pursh $(1,2)$ AGS, BCN, CHIH, CHIS, DGO, GTO, HGO, JAL, MEX, MICH, OAX, PUE, QRO, SLP, SON, VER, ZAC.

\section{CERATOPHYLLACEAE}

Ceratophyllum demersum L. (1, 2) BCN, BCS, CAM, CHIS, COAH, COL, DF, DGO, GRO, HGO, JAL, MEX, MICH, MOR, OAX, PUE, QRO, SLP, SIN, SON, TAB, TAMS, TLAX, VER.

Ceratophyllum muricatum Cham. $(1,2)$ CHIS, COL, DF, GRO, JAL, OAX, TAB, VER.

\section{COMBRETACEAE}

Laguncularia racemosa (L.) C.F. Gaertn. (1) BCS, CAM, CHIS, COL, GRO, JAL, MICH, NAY, OAX, QROO, SIN, SON, TAB, TAMS, VER, YUC.

\section{CRASSULACEAE}

Crassula saginoides (Maxim.) M. Bywater \& Wickens $(1,2)$ AGS, BCS, DF, GTO, HGO, JAL, MEX, MICH, MOR, QRO.

\section{ELATINACEAE}

*Bergia capensis L. (1) OAX.

Bergia texana (Hook.) Seub. ex Walp. (1) BCN, BCS, NL, SIN, SON, TAMS.

Elatine brachysperma A. Gray (2) AGS, BCN, CHIS, GTO, HGO, JAL, MEX, MICH, OAX, QRO, SLP, SON.

Elatine californica A. Gray (2) BCN.

\section{EUPHORBIACEAE}

Phyllanthus fluitans Benth. ex Müll. Arg. (1) TAB.

\section{HALORAGACEAE}

*Myriophyllum aquaticum (Vell.) Verdc. $(1,2)$ CHIS, DF, GTO, HGO, JAL, MEX, MICH, MOR, OAX, SON, TAMS, ZAC.

Myriophyllum farwellii Morong (2) MICH.

Myriophyllum heterophyllum Michx. $(1,2)$ CAM, CHIS, HGO, JAL, MEX, MICH, MOR, VER. 
Apéndice. Continuación.

Myriophyllum hippuroides Nutt. ex Torr. \& A. Gray $(1,2) \mathrm{CHIH}, \mathrm{MEX}, \mathrm{MICH}, \mathrm{MOR}, \mathrm{QRO}$, TAMS.

Myriophyllum pinnatum (Walter) Britton, Sterns \& Poggenb. (2) CHIH, MICH.

Myriophyllum quitense Kunth (2) MEX.

Myriophyllum sibiricum Kom. (1) BCN, CHIH, SON.

Proserpinaca palustris L. (2) CHIS, VER.

Proserpinaca pectinata Lam. (1) TAB.

\section{LENTIBULARIACEAE}

Utricularia foliosa L. (1, 2) CAM, CHIS, GRO, GTO, JAL, OAX, QRO, QROO, TAB, TAMS, VER.

Utricularia gibba L. (1, 2) CAM, CHIH, CHIS, COAH, DF, DGO, GRO, GTO, HGO, JAL, MEX, MICH, MOR, PUE, QRO, QROO, SLP, SON, TAB, TAMS, VER, YUC, ZAC. Utricularia hydrocarpa Vahl (1) TAB.

Utricularia macrorhiza Leconte (2) BCS, COAH, DF, MEX, MICH, PUE.

Utricularia perversa P. Taylor (2) CHIH, GTO, HGO, JAL, MEX, OAX, QRO.

Utricularia purpurea Walter (1) QROO, TAB.

Utricularia radiata Small (1) TAB.

\section{LOBELIACEAE}

Lobelia purpusii Brandegee $(1,2)$ CHIS, DGO, PUE, QRO, SLP, TAMS, VER.

\section{LYTHRACEAE}

Rotala mexicana Schltdl. \& Cham. $(1,2)$ CAM, CHIH, CHIS, GRO, QRO, VER.

\section{MENYANTHACEAE}

Nymphoides fallax Ornduff $(1,2)$ AGS, CHIH, CHIS, DF, DGO, GTO, HGO, JAL, MEX, MICH, NAY, OAX, PUE, QRO, TLAX, VER, ZAC.

Nymphoides indica (L.) Kuntze $(1,2)$ CAM, CHIS, COL, GRO, HGO, JAL, MICH, NAY, QRO, QROO, TAB, TAMS, VER, YUC.

\section{MIMOSACEAE}

Neptunia natans (L. f.) Druce (1) CAM, CHIS, GRO, JAL, MICH, MOR, NAY, OAX, SIN, TAB, TAMS, VER.

NELUMBONACEAE

Nelumbo lutea Willd. (1) CAM, CHIS, JAL, NAY, QROO, TAB, TAMS, VER.

NYMPHAEACEAE

Nuphar advena (Aiton) W.T. Aiton (1) COAH, NL, TAMS, VER.

Nymphaea amazonum Mart. \& Zucc. (1) CAM, CHIS, OAX, TAB, TAMS. 
Apéndice. Continuación.

Nymphaea ampla (Salisb.) DC. (1, 2) CAM, CHIS, COAH, COL, GRO, JAL, MICH, NAY, NL, OAX, PUE, QRO, QROO, SLP, SON, TAB, TAMS, VER, YUC.

*Nymphaea capensis Thunb. (1) QROO.

Nymphaea conardii Wiersema (1) CAM, CHIS, OAX, TAB, VER.

Nymphaea elegans Hook. (1) BCS, GRO, HGO, JAL, MOR, NAY, NL, OAX, QROO, SIN, SON, TAMS, VER.

Nymphaea gracilis Zucc. (2) AGS, DF, DGO, GTO, JAL, MEX, MICH, OAX.

Nymphaea jamesoniana Planch. (1) CAM, CHIS, GRO, JAL, OAX, QROO, TAB, TAMS, VER.

Nymphaea mexicana Zucc. $(1,2)$ DF, HGO, MEX, MICH, NL, OAX, SON, TAMS, VER.

Nymphaea novogranatensis Wiersema (1) OAX.

Nymphaea odorata Aiton (2) DF, DGO, HGO, MEX, MICH, SON, VER.

Nymphaea prolifera Wiersema (1) TAB, VER.

Nymphaea pulchella DC. $(1,2)$ CAM, GRO, JAL, MICH, MOR, NAY, OAX, PUE, QROO, TAB, YUC, ZAC.

\section{ONAGRACEAE}

Ludwigia adscendens (L.) H. Hara $(1,2) \mathrm{HGO}$, JAL, MICH, TAMS, VER.

Ludwigia helminthorrhiza (Mart.) H. Hara (1) CHIS, GRO, JAL, NAY, OAX, TAB.

Ludwigia inclinata (L. f.) M. Gómez (1) CHIS, GRO, OAX, TAB, VER.

Ludwigia palustris (L.) Elliott $(1,2)$ CHIH, CHIS, DF, DGO, GRO, HGO, JAL, MEX, MICH, MOR, NL, QROO, SIN, SLP, SON, TAB, TAMS, VER.

Ludwigia peploides (Kunth) P.H. Raven (1, 2) AGS, BCN, BCS, CAM, CHIH, CHIS, COL, DF, DGO, GRO, GTO, HGO, JAL, MEX, MICH, MOR, NAY, OAX, PUE, QRO, QROO, SIN, SLP, SON, TAB, TAMS, TLAX, VER, ZAC.

Ludwigia repens J.R. Forst. $(1,2)$ CHIH, COAH, JAL, MEX, MICH, MOR, PUE, QRO, SLP, TAMS, VER, ZAC.

Ludwigia sedoides (Bonpl.) H. Hara (1) CAM, CHIS, TAB, VER, AGS.

Ludwigia torulosa (Arn.) H. Hara (1) TAB, VER.

\section{PODOSTEMACEAE}

Marathrum minutiflorum Engl. $(1,2)$ CHIS, OAX, TAB, VER.

Marathrum rubrum Novelo \& C. T. Philbrick (1) GRO, JAL.

Marathrum schiedeanum Cham. (1) COL, CHIS, GRO, JAL, MICH, MOR, NAY, OAX, VER. Marathrum tenue Liebm. $(1,2)$ CHIS, MOR, OAX, PUE, VER.

Oserya coulteriana Tul. $(1,2)$ BCS, CHIH, COL, GRO, JAL, MICH, MOR, NAY, SIN, SON, TAMS.

Oserya longifolia Novelo \& C. T. Philbrick (1) JAL.

Podostemum rutifolium Warm. $(1,2)$ OAX, PUE, TAB, VER.

Tristicha trifaria (Bory ex Willd.) Spreng. (1, 2) CHIS, COL, GRO, HGO, JAL, MEX, MOR, NAY, OAX, PUE, SLP, TAB, TAMS, VER, ZAC. 
Apéndice. Continuación.

Vanroyenella plumosa Novelo \& C. T. Philbrick (1) JAL, OAX.

POLYGONACEAE

Polygonum acuminatum Kunth $(1,2)$ CAM, CHIS, GRO, MOR, QROO, TAB, TAMS, VER.

*Polygonum amphibium L. (2) BCN, BCS, CHIH, DF, HGO, MEX, MICH, MOR, PUE, SON.

\section{RANUNCULACEAE}

Ranunculus cymbalaria Pursh $(1,2)$ AGS, BCN, CHIH, COAH, DF, DGO, GTO, HGO, MEX, MICH, MOR, PUE, QRO, SLP, TLAX, VER.

Ranunculus flagelliformis Sm. (2) CHIS, HGO, OAX, PUE.

Ranunculus hydrocharoides A. Gray (2) BCN, BCS, CHIH, CHIS, DF, DGO, GTO, HGO, JAL, MEX, MICH, MOR, QRO, SLP, SON, TLAX, VER, ZAC.

Ranunculus trichophyllus Chaix ex Vill. (2) BCN, CHIH, COAH, DF, DGO, HGO, MEX, MOR, SON, TLAX, VER.

RHIZOPHORACEAE

Rhizophora mangle L. (1) BCN, BCS, CAM, CHIS, COL, GRO, JAL, MICH, NAY, OAX, QROO, SIN, SON, TAB, TAMS, VER, YUC.

SCROPHULARIACEAE

Benjaminia reflexa (Benth.) D'Arcy (1) QROO, TAB, VER.

SOLANACEAE

Solanum tampicense Dunal (1) CAM, CHIS, GRO, JAL, NAY, OAX, TAB, TAMS, VER, YUC.

\section{SPHENOCLEACEAE}

*Sphenoclea zeylanica Gaertn. (1) CAM, CHIS, COL, GRO, OAX, TAB, TAMS, VER. 